\title{
Lattice-Boltzmann simulation of grid-generated turbulence
}

\author{
By L. DJENIDI \\ Discipline of Mechanical Engineering, University of Newcastle, Newcastle, 2308 NSW, Australia
}

(Received 23 November 2004 and in revised form 26 September 2005)

The lattice-Boltzmann method (LBM) is used to carry out a direct numerical simulation (DNS) of grid-generated turbulence with the view to improve comparison between experimental and numerical results on approximate isotropic turbulence. The grid is made up of four by four floating flat square elements in an aligned arrangement. The Reynolds number based on the Taylor microscale is about 40 at a distance of 70 times the separation between the elements downstream of the grid; this value is comparable to that of many experiments.

While the results compare relatively well with existing experimental data on grid turbulence (grid made up of bars), they highlight the importance of the mesh resolution of the simulation and computational domain size in the decay of turbulence. For example, while a power-law decay could be identified, at least over a short distance, its decay exponent proves to be difficult to determine with good accuracy. This points out the need for simulations (and perhaps experiments too) where all scales are properly solved before conclusions can be drawn.

\section{Introduction}

Homogeneous and isotropic turbulence is the simplest form of turbulence, and is experimentally studied when generated by a grid, where a fluid flows uniformly through a grid, usually made of either square or round vertical and horizontal bars. The grid is characterized by the diameter of the bars, $D$, and their spacing, $M$, and/or the solidity, $\sigma$. The flow parameters governing the turbulence are the mesh and Taylormicroscale turbulence Reynolds numbers, $R_{M}\left(=U_{0} M / v\right)$ and $R_{\lambda}(=u \lambda / v$; the latter is sometimes called the turbulence Reynolds number), respectively. In these expressions, $U_{0}$ is the mean velocity, $v$ is the kinematic viscosity of the fluid, $u$ a velocity scale characteristic of turbulence, and $\lambda$ the Taylor longitudinal microscale. Apart from the fact that grid-generated turbulence is only in an approximately isotropic state, as all studies have shown, the question of the universality of turbulence in such a flow arises as the initial conditions may affect its decay (George et al. 1992; George 2001). While an experiment is currently in progress to investigate these latter effects (Lavoie et al. 2005; Lavoie, Antonia \& Djenidi 2006), it is experimentally impossible to study all possible grid geometries. It is clear that only direct numerical simulation (DNS) can help in this task.

So far, DNS of isotropic turbulence has been carried out in a cubic box of side $L$, with periodic conditions in the three directions, and for either decaying or forced turbulence. While the use of a periodic cubic box improves the establishment of an homogeneous and isotropic state (the ideal would be to carry out DNS in an unbounded and non-periodic domain), the simulations are not problem free. Decaying turbulence 
is generated with an initial spectrum and the DNS solves for the subsequent decay. Not does only this procedure generate relatively low values of $R_{\lambda}$, but it may also raise the question of the choice of the initial spectrum form and its effect on the decay of the turbulence. Larger $R_{\lambda}$ can be achieved when a (random) forcing term is introduced into the Navier-Stokes equations; turbulence is maintained and reaches a steady state. Unfortunately in this case, the results are dependent on the statistics of the forcing. It should be pointed out that forced turbulence may not be relevant to the problem of decaying turbulence in the sense that the term $\mathrm{d} E(k, t) / \mathrm{d} t$ (where $E(k, t)$ is the turbulence energy, $k$ is the wavenumber and $t$ the time) is statistically zero in the energy balance equation when turbulence is forced. Since the focus here is on the decaying turbulence, we will not discuss further the case of forced turbulence.

Comparing results between box turbulence and grid-generated turbulence may not be appropriate because of the different types of turbulence generation. In that respect, it would be better to have numerical and experimental data on turbulence generated in a similar fashion. Thus recent experimental attempts to generated isotropic turbulence in a box (Birouk, Sarh \& Gokalp 2003; Hwang \& Eaton 2003) are interesting. However, the effects of the initial conditions may still be of concern: while turbulence spectra are used to initialize the DNS, fans or jets are utilized to generate turbulence in the box experiment. This may be a source of discrepancies when comparing the results. In that respect, DNS of grid-generated turbulence where the grid is included in the computation domain (to generate turbulence) seems to be appropriate. Such simulations would make the comparison of the results between computation and measurements more relevant. Note that the issue raised since George's (1992) work as to whether initial conditions can persist in turbulence is yet to be properly addressed. Such an issue concerns both DNS and experiments.

There is currently no reported DNS of grid-generated turbulence, whether the grid is included or not in the computational domain, for direct comparison with experimental results. Thus, this paper reports the first DNS of grid-generated turbulence and an exploratory study. The DNS is carried out through the lattice-Boltzmann method (LBM) rather then solving the Navier-Stokes equations and the reasons for this are given in $\S 2$ where the numerical procedure is detailed. Results are presented and discussed in $\S 3$.

\section{Numerical procedure}

\subsection{The lattice-Boltzmann method}

The grid-generated turbulence is simulated using the lattice-Boltzmann method, which is based on kinetic theory. Rather than solving the governing fluid equations (NavierStokes equations), the LBM solves the Boltzmann equation on a lattice. The basic idea of the LBM is to construct a simplified kinetic model that incorporates the essential physics of microscopic average properties, which obey the desired (macroscopic) Navier-Stokes equations (Frisch, Hasslacher \& Pomeau 1984). With a sufficient amount of symmetry of the lattice, the LBM implicitly solves these equations with second-order accuracy. For the present calculations, each computational node consists of a three-dimensional lattice composed of 18 moving particles and a rest particle (lattice model D3Q19, figure 1). The spacings in the three directions between each node are $\Delta x, \Delta y$, and $\Delta z$.

The Boltzmann equation is discretized on that lattice and results in the latticeBoltzmann equation, which governs the time and space variations of the single-particle 


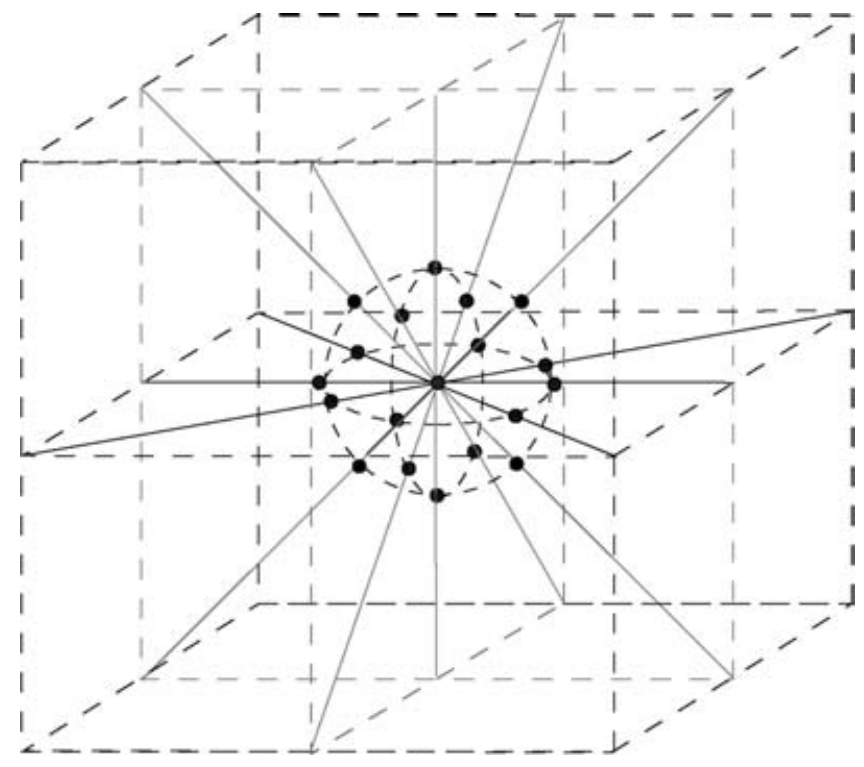

FIGURE 1. D3Q19 square lattice model.

distribution $f_{i}(\boldsymbol{x}, t)$ at the lattice site $\boldsymbol{x}$ :

$$
f_{i}\left(\boldsymbol{x}+e_{i} \Delta t, t+\Delta t\right)-f_{i}(\boldsymbol{x}, t)=-\frac{\Delta t}{\tau}\left(f_{i}(\boldsymbol{x}, t)-f_{i}^{e q}(\boldsymbol{x}, t)\right), i=0,1, \ldots, 18,
$$

where $\tau$ is the relaxation time, $\Delta t$ the time step, $\boldsymbol{e}_{i}(=\Delta x / \Delta t)$ is the particle velocity in the $i$-direction and $f_{i}^{e q}$ is the equilibrium single-particle distribution:

$$
f_{i}^{e q}=\rho \omega_{i}\left(1+3\left(\boldsymbol{e}_{i} \cdot \boldsymbol{u}\right)+\frac{9}{2}\left(\boldsymbol{e}_{i} \cdot \boldsymbol{u}\right)^{2}-\frac{3}{2} u^{2}\right)
$$

where $\rho\left(=\sum_{i} f_{i}\right)$ is the fluid density, $\boldsymbol{u}\left(\rho \boldsymbol{u}=\sum_{i} f_{i} \boldsymbol{e}_{i}\right)$ is the local fluid velocity and $\omega_{i}$ are the corresponding weights $\left(\omega_{i}=1 / 3\right.$ for $i=0,1 / 18$ for $i=1$ to 6 , and $1 / 36$ for $i=7$ to $18 ; i=0$ corresponds to the rest particle in the centre of the cubic lattice, $i=1, \ldots, 6$, correspond to the particles on the axis aligned with $x, y$ and $z$, and $i=7 \ldots, 18$, are related to the particles on the diagonal directions.) The relaxation time is related to the kinematic viscosity of the fluid via the relation

$$
v=\frac{2 \tau-1}{6} \text {. }
$$

In the above equations $\Delta t=\Delta x=\Delta y=\Delta z=1$. With this choice, $\left|\boldsymbol{e}_{i}\right|=1$, for $i=1$ to 6 , and $\sqrt{2}$ for $i=7$ to 18 ; note that $\left|\boldsymbol{e}_{0}\right|=0$, and the dimensional variables such as, $u, v$, and $\rho$ are expressed in lattice units. Note that $v$ is estimated once the Reynolds number is fixed $\left(v=U_{0} M / R_{M}\right)$. The relation between the lattice units and the real dimensions can be obtained by writing $L_{L B M}=N \times \Delta x_{L B M}$ and $L_{\text {real }}=N \times \Delta x_{\text {real }}$, which yields $\Delta x_{\text {real }}=\left(L_{\text {real }} / L_{L B M}\right) \Delta x_{L B M}$ where $L_{L B M}$ and $\Delta x_{L B M}$ are a length scale and the space step in lattice units and $L_{\text {real }}$ and $\Delta x_{\text {real }}$ are a length scale and the space step in real dimensions; $N$ is the number of mesh points of in the $x$-direction.

The left-hand side of (2.1) is the so-called streaming operation, which means that the particles move to the nearest neighbours in their velocity directions (i.e. they radiate from the centre of the lattice in their velocity direction). The right-hand side is the collision term, here modelled by the BGK collision operator, which describes the redistribution of the particles at each node (for more detail see, for example, Chen \& 


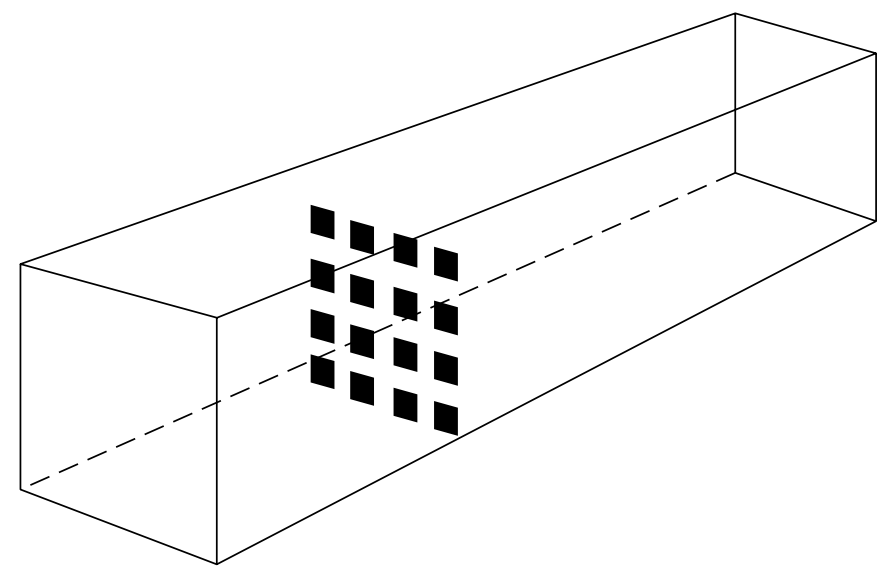

FIGURE 2. Sketch (not to scale) of the computational domain with the grid.

Doolen 1998). Thus (2.1) is solved according to these two operations: collision and streaming. The collision step is described by

$$
f_{i}^{n e w}(\boldsymbol{x}, t)=f_{i}(\boldsymbol{x}, t)-\frac{\Delta t}{\tau}\left(f_{i}(\boldsymbol{x}, t)-f_{i}^{e q}(\boldsymbol{x}, t)\right),
$$

where the $f_{i}^{e q}$ are calculated using (2.2). The streaming is described by

$$
f_{i}\left(\boldsymbol{x}+e_{i} \Delta t, t+\Delta t\right)=f_{i}^{n e w}(\boldsymbol{x}, t) .
$$

The collisions are entirely local, making the LBM efficiently parallelized. At time $t$, the particle distributions are updated based on (2.4); then, at time $t+\Delta t$, the particles propagate according to $(2.5)$.

The choice of the LBM over the classical resolution of the Navier-Stokes equations for the present simulations was motivated by its two important and practical advantages: (i) extreme ease of implementation of complex solid surfaces, and (ii) the local nature of the collision renders the parallelization of any LBM code quite natural and simple.

\subsection{The computational details}

The computational uniform Cartesian mesh consists of $690 \times 73 \times 73$ mesh points (figure 2) with $\Delta x=\Delta y=\Delta z=1$ ( $x$ is the longitudinal direction and $y$ and $z$ the lateral directions). The turbulence-generating grid (placed at the $x$-node of 50) is made up of $4 \times 4$ floating flat square elements in an aligned arrangement (figure 2). Each element is represented by $9 \times 9$ mesh points and the spacing $(M)$ between the elements is 9 mesh points, yielding a grid solidity of 0.25 . The downstream distance extends to $x / M=71$, where the origin of $x$ is taken at the grid location. Note that the same distance would require a far too large number of mesh points if a turbulencegenerating grid with the equivalent solidity made of bars were to be used. This is the main reason why the square elements were chosen.

Periodic conditions are applied in the $y$ - and $z$-directions. At the inlet a uniform velocity $\left(U_{0}=0.05\right.$, and $\left.V_{0}=W_{0}=0\right)$ is imposed, and a zero gradient is applied at the outlet; a convective boundary condition was also tried at the outlet and no significant difference was observed in the results. To simulate the no-slip condition at the grid elements, a bounce-back boundary is used; when a particle reaches the wall it is reflected back in the direction from which it arrived. 
The Reynolds number, $R_{M}$, is about 1600 . This is a relatively small value, which allows a reasonably good grid resolution, varying from about $4 \eta$ at $x / M=20$ to $2 \eta$ at $x / M=60$ where $\eta$ is the Kolmogorov length scale (the various scales will be discussed further later). It should be noted the small value of $R_{M}$ requires caution when discussing the results; when $R_{M}$ is low the condition for incompressibility is still satisfied in the present simulation as the Mach number $U / c=0.028$ ( $c$ is the speed of sound and is equal to $1 / \sqrt{3}$ in lattice units for the present lattice molecule).

In order to reduce the transient period, a noise was superimposed on the initial velocity for a short time. The 'steady' state solution is obtained after 10000 iterations. After only the 30000 th iteration is the first velocity field saved. Subsequently, 74 velocity fields are recorded, each separated by 1000 iterations (about five times $D / U$, where $D$ is the element size) to ensure that two consecutive fields are uncorrelated.

Preliminary calculation revealed that instabilities occured where the magnitude of the local strain rate

$$
\Pi_{\alpha \beta}=\frac{1}{2}\left(\frac{\partial u_{\alpha}}{\partial x_{\beta}}+\frac{\partial u_{\beta}}{\partial x_{\alpha}}\right)=\sum_{1} e_{i \alpha} e_{i \beta}\left(f_{i}-f_{i}^{e q}\right)
$$

is large. The instabilities occurred mainly around the turbulence-generating grid elements. To help dissipate these instabilities, a large-eddy simulation (LES) scheme is introduced:

$$
v_{\text {total }}=v+v_{t}
$$

where $v_{t}$ is the turbulent viscosity. The Smagorinsky scheme is used in the present simulation. Thus, following Hou et al. (1996), we have

$$
v_{\text {total }}=v+(C \Delta)^{2}|S|
$$

with $C=0.1, \Delta=\Delta x$ and

$$
|S|=\frac{\sqrt{v^{2}+18(C \Delta)^{2}\left(\Pi_{i j} \Pi_{i j}\right)^{1 / 2}}-v}{\left.6(C \Delta)^{2}\right)} .
$$

In terms of the time relaxation the LES yields

$$
\left.\tau_{\text {total }}=3\left(v^{2}+(C \Delta)^{2}\right)|S|\right)+\frac{1}{2} .
$$

It is important to emphasize that since the LES is used merely as a dissipative scheme for the simulations, no attempt is made to investigate various LES models and their effects on the simulations. Furthemore, since the LES acts at a subgrid level less than $\Delta x$, it is believed that the LES model should not significantly change the results concerning motions whose scales are larger than a few times the Kolmogorov length scale.

The calculations were carried out on a dual processor $\left(2 \times 2.4 \mathrm{GH}_{\mathrm{z}}\right) \mathrm{HP}$ workstation. The collision and streaming steps were parallelized using the OpenMP procedure. During these steps, each processor worked on half the computational domain.

\section{Results}

\subsection{Numerical visualizations}

Figures $3(a)$ and $3(b)$ show the velocity fields in the $(x, y)$ - and $(x, z)$-planes for $0<x / M<20$ behind two perpendicular rows of grid elements (figure 3(c) shows the same field as in figure $3(b)$ but with the mean velocity subtracted). For comparison, 

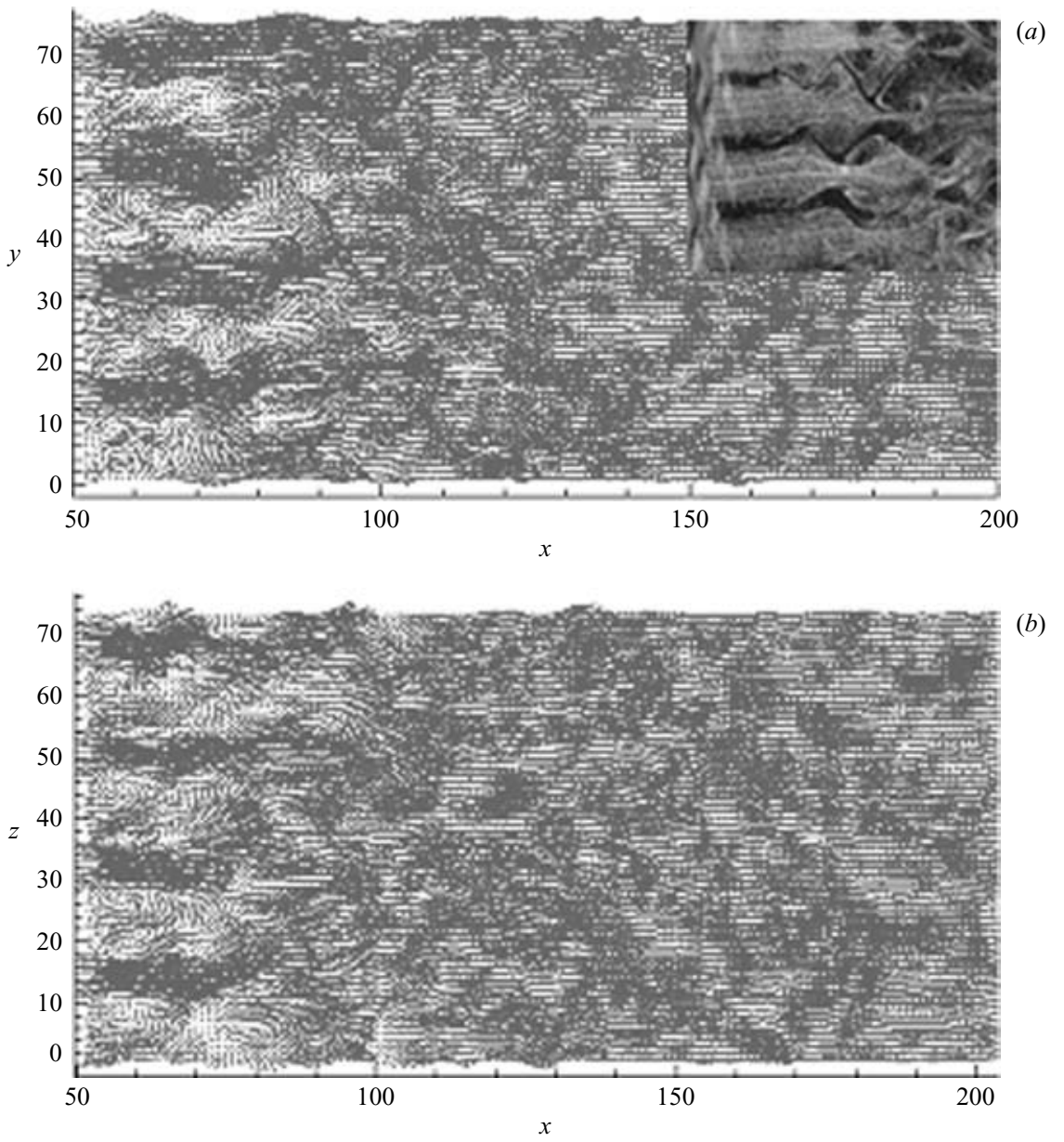

(b)

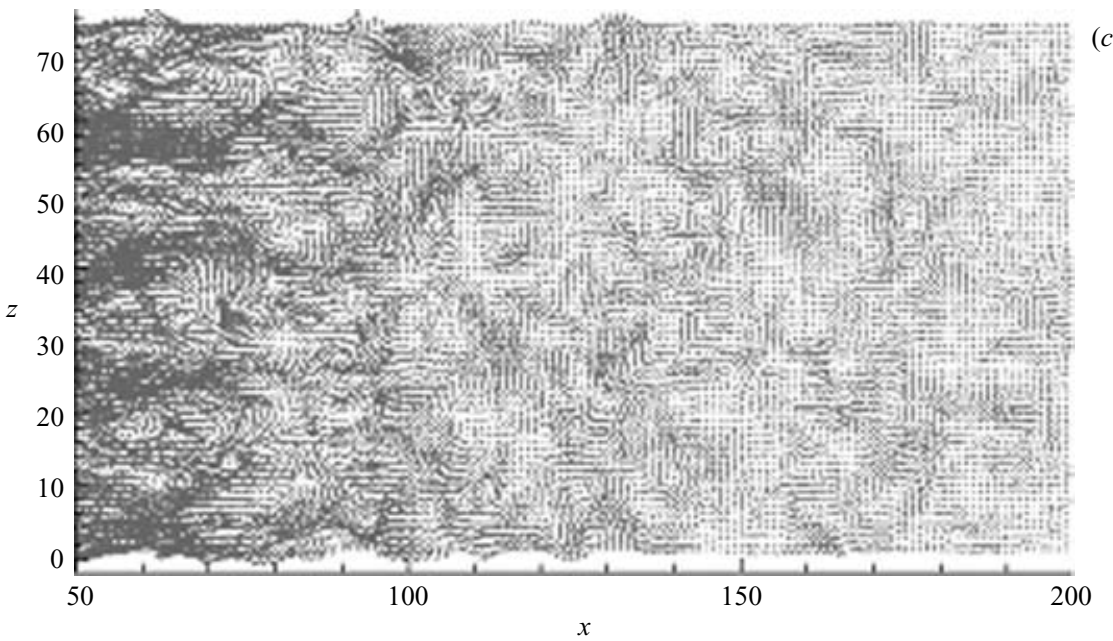

FIGURE 3. Velocity field behind the grid. $(a)(x, y)$-plane; $(b)(x, z)$-plane; $(c)(x, z)$-plane and with the mean velocity subtracted. The inset in $(a)$ is taken from (Lavoie et al. 2006). 


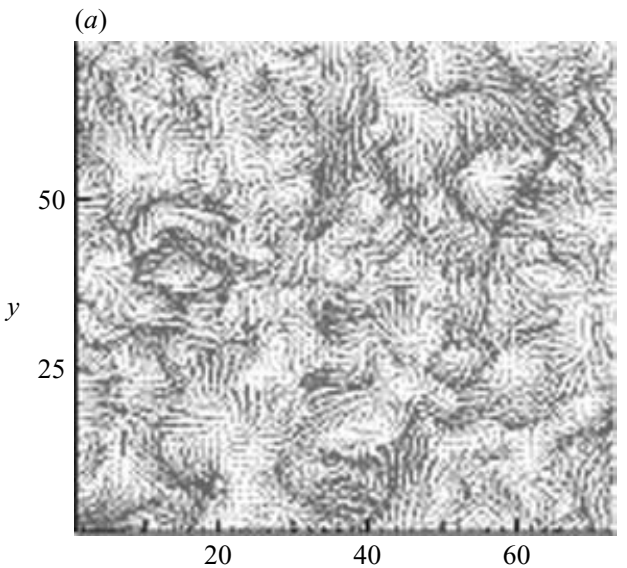

(b)

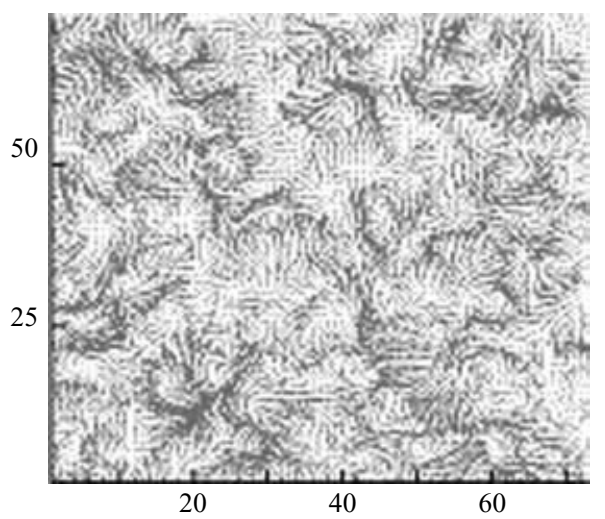

(c)

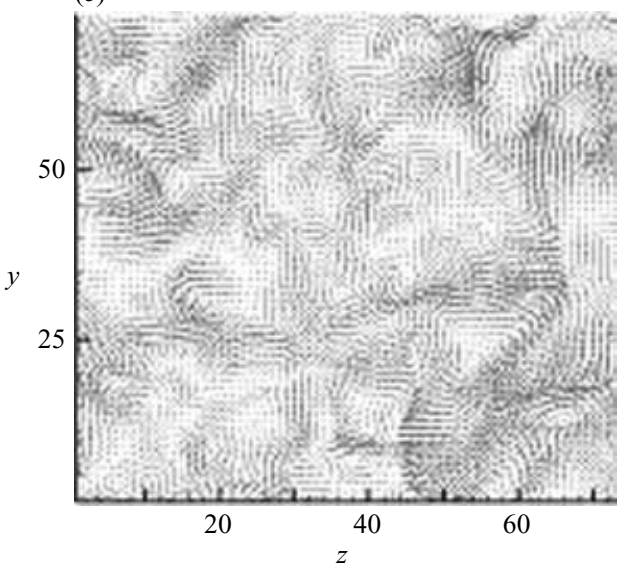

(d)

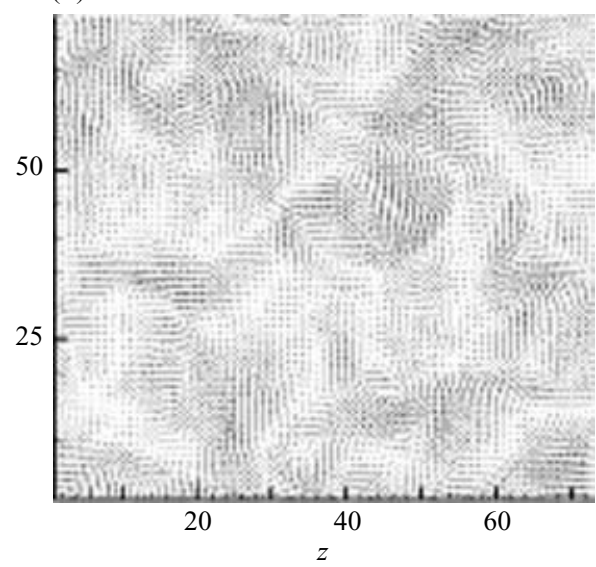

FIGURE 4. Velocity field behind the grid in the $(z, y)$-plane. (a) $x / M=5 ;(b) x / M=20$; (c) $x / M=40 ;(d) x / M=60$.

the inset on figure 3(a) shows a flow visualization sequence taken behind a bi-planar grid (vertical and horizontal bars, $M=4 D$ ), visible on the left side, made of square bars. The flow visualization was performed in a small water tunnel at $R_{M}=350$ (Lavoie et al. 2006). The visual field covers the region $0<x / M<3-4$.

There is a similarity between the calculated velocity fields and the picture, even though the grid geometry differs between them. Coherent vortical structures are clearly visible behind the grid in the computational and experimental results. The structures, shed by the grid elements, are convected downstream, merge relatively quickly, and, through their interaction, generate turbulence, which reaches a maximum at a streamwise distance equal to a few times the mesh size. Owing to the grid geometry difference, the vortical structures shed by the square elements are different from those shed by the bars. Thus, one could investigate to what extent this difference, which is analogous to different initial conditions in homogeneous decaying turbulence, affects the decay of turbulence.

Figure 3(c) suggests that turbulence, produced in the region $0<x / M<5$ through the interaction of these structures, is high in the region $5<x / M<10$ and decays quite rapidly as $x / M$ increases, which is illustrated in figure 4 , showing the velocity field in 

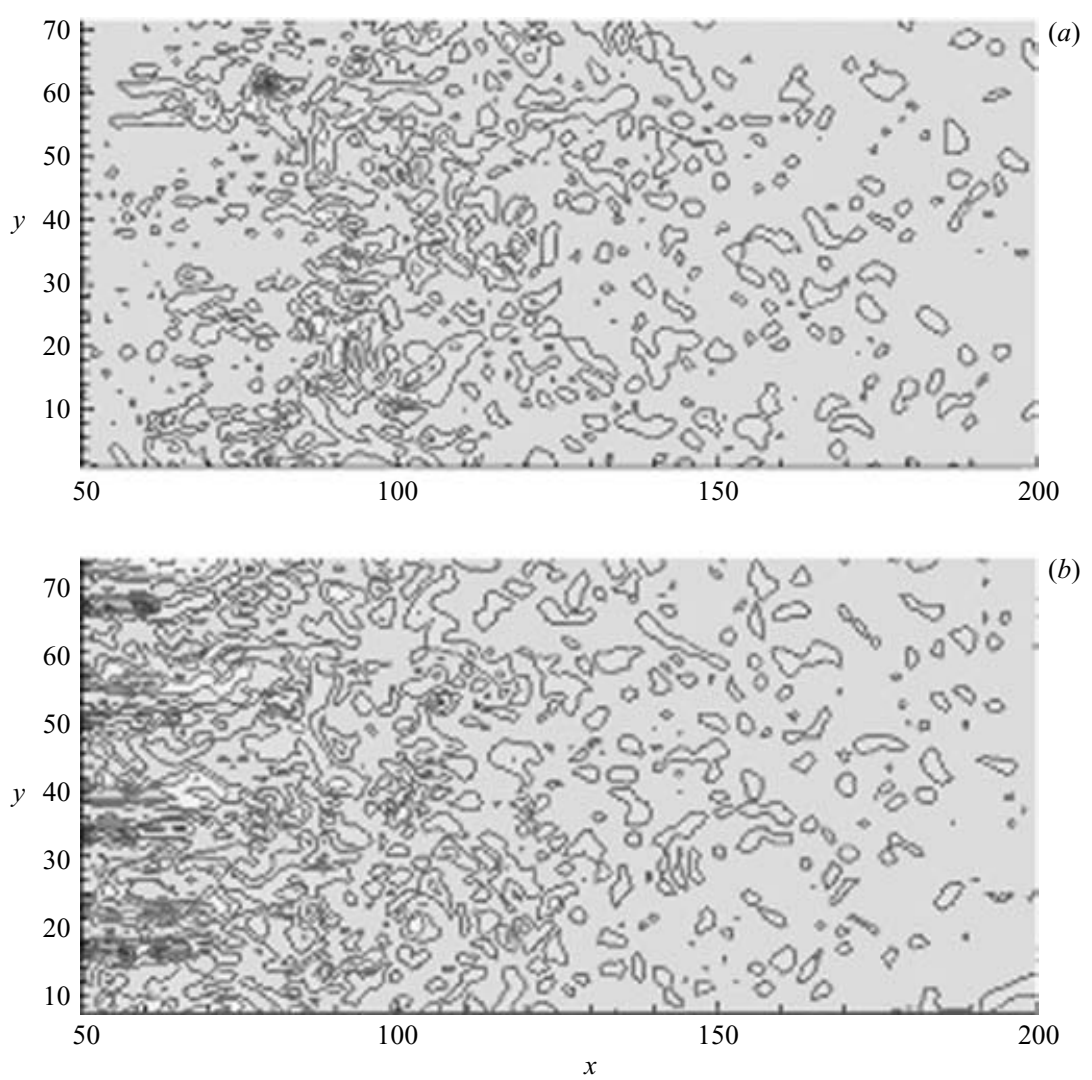

FIGURE 5. Iso-surface of the spanwise component of the vorticity in the $(x, y)$-planes $(a)$ between two rows of grid elements, $(b)$ behind a row of grid elements.

the $(z, y)$-plane at $x / M=5,20,40$ and 60. This figure clearly indicates that the $v$ and $w$ fields are statistically similar (as expected due to the symmetry of the problem) and thus the usual assumption $v^{\prime 2}=w^{\prime 2}$ ( $v$ and $w$ are velocity fluctuations in the $y$ - and $z$-directions, respectively, and the prime denotes the root mean square) is justified, at least for symmmetrical grids such as used here. Notice the decrease in the coherence of the vortical structures by the downstream distance, accompanied by an apparent growth of the length scale.

Figure 3 also reveals that the velocity field is likely to be highly inhomogeneous behind the grid to some distance downstream. This is clearly visible in the iso-contours of one transversal component, $\omega_{z}$, of the vorticity, taken in the $(x, y)$-plane between two rows of grid elements and behind one row, shown in figure 5. The map of $\omega_{z}$ indicates that relatively strong coherent vortical structures are generated around the grid elements and interact quickly as indicated by the division of the iso-contours. Considering that a similar mechanism occurs in the $(x, z)$-plane, one can easily infer the relatively strong three-dimensional character of the flow behind the grid resulting in a highly inhomogeneous velocity field. This feature is also visible in figure 6 , where iso-surfaces of the streamwise component, $\omega_{x}$, of the vorticity are represented. The view in the $(z, y)$-plane in figure 6 shows that $\omega_{x}$ is mainly located behind each grid element, while the three-dimensional view indicates that $\omega_{x}$ is produced slightly 


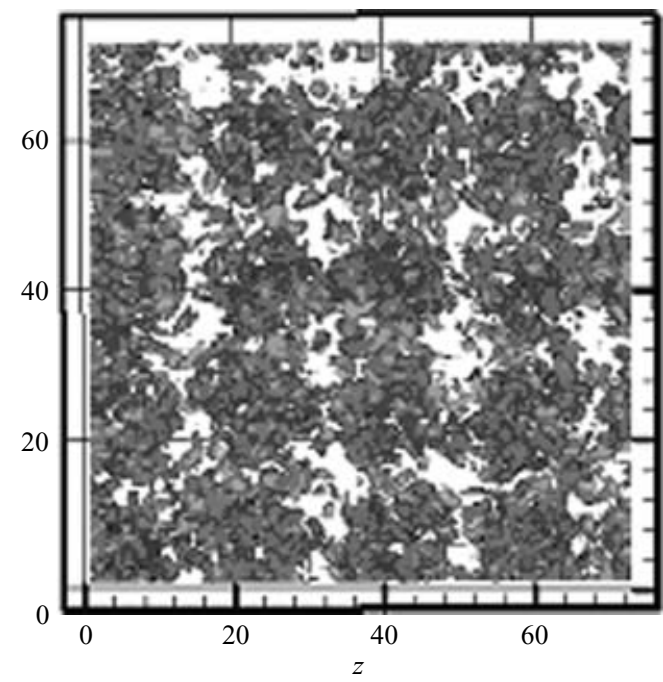

(a)

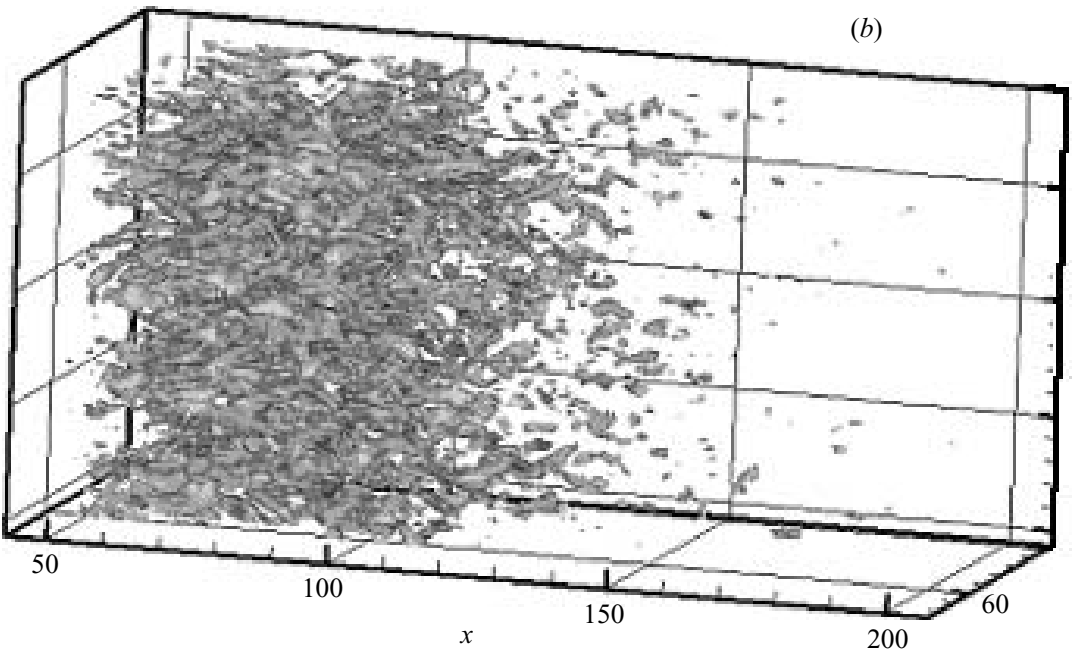

FiguRE 6. Iso-surface of the streamwise component of the vorticity $(a)$ in the $(z, y)$ and $(b)$ in a three-dimensional view $\left(\omega_{x}=-0.015,0.015\right)$.

downstream of the grid than $\omega_{z}$ and $\omega_{y}$ (see figure 5). This is clearly related to the grid geometry. The fact that the grid is made of isolated floating elements results in more production of the transverse components than the streamwise component. As the $(z, y)$ and the 3-D views in figure 6 represent the same $\omega_{x}$ field, they reveal that the upstream conditions are still felt at $x / M$ of about 6 , as illustrated by the relatively regular pattern of the packets of $\omega_{x}$ in the plane $(z, y)$.

Finally, iso-surfaces of $\omega^{2}$, the square of the instantaneous vorticity, are shown in figure 7. Around the grid elements the iso-surfaces are highly coherent and have length scale of the order of magnitude of the element size (note their cylindrical-like form). However, at about $x=M$, they start breaking up due to their interaction, and then burst into a large number of smaller surfaces. Thus, the turbulence production mechanism for the present grid appears to consist of the generation of vorticity 'tubes', 


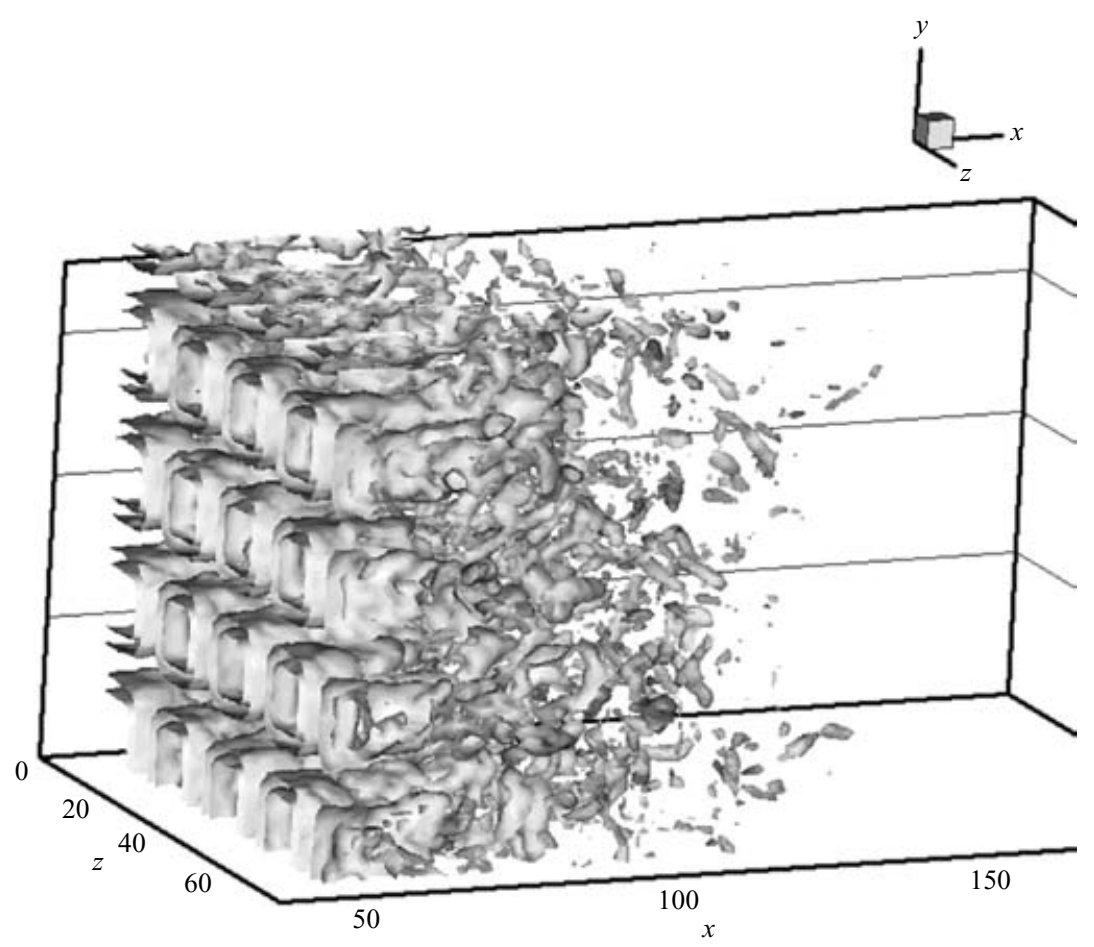

FIGURE 7. Iso-surfaces of $\omega^{2}$ behind the grid.

followed by their strong interaction. The whole process takes place over a distance of $x / M$ about 5 .

\subsection{Statistics}

Figure 8 shows the decay of $u^{\prime 2} / U_{0}^{2}, v^{\prime 2} / U_{0}^{2}, w^{\prime 2} / U_{0}^{2}$ and $q^{\prime 2} / U_{0}^{2}=\left(u^{\prime 2}+v^{\prime 2}+w^{\prime 2}\right) / U_{0}^{2}$, averaged in time and space ( $y$ - and $z$-directions). As noted in the previous section, $v^{\prime 2}$ and $w^{\prime 2}$ are identical and only one of the two velocity components ( $v$ or $w$ ) will be considered for the statistical analysis. The experimental data of Lavoie et al. (2005; $\left.R_{M}=10000\right)$ are also shown for comparison.

The calculated ratios, while being higher in magnitude than the experimental data, reproduce the expected result of grid-turbulence decay, which is $u^{\prime 2}>v^{\prime 2}$. Just behind the grid, $u^{\prime 2}$ is much stronger than $v^{\prime 2}$ and $w^{\prime 2}$ indicating that the longitudinal component of the velocity fluctuations receives more energy than the transverse ones. Notice that the location of the maximum of $u^{\prime 2}$ is at $x / M=1$, while the maximum of $v^{\prime 2}$ is reached only at $x / M=2.9$. This maximum shift results in a perceptible bump in the $q^{\prime 2}$ distribution at $x / M$ of about 6-8. This bump is also observed in DNS data of decaying homogeneous isotropic box turbulence in a periodic box (Antonia $\&$ Orlandi 2004). It would be of interest to compare the variations of $u^{\prime 2}$ and $v^{\prime 2}$ in the region $x / M<3$ with that of a grid made of bars. The difference between $u^{\prime 2}$ and $v^{\prime 2}$, when the latter has started to decay, is maintained throughout the energy decay.

The distributions appear to be consistent with a power-law decay $\left(q^{\prime 2} \propto((x-\right.$ $\left.\left.x_{0}\right) / M\right)^{n}$ or $u^{\prime 2} \propto\left(\left(x-x_{0}\right) / M\right)^{n}, x_{0}$ is a virtual origin) for $x / M>40$. A linear curve fit to the $q^{\prime 2}$ distribution for $x / M>40$, yields a decay exponent $n$ of about -1.53 . However, when plotted on a larger scale, none of the four curves presents a clear straight line region (this is also observed with the data of Lavoie et al. 2005). This 


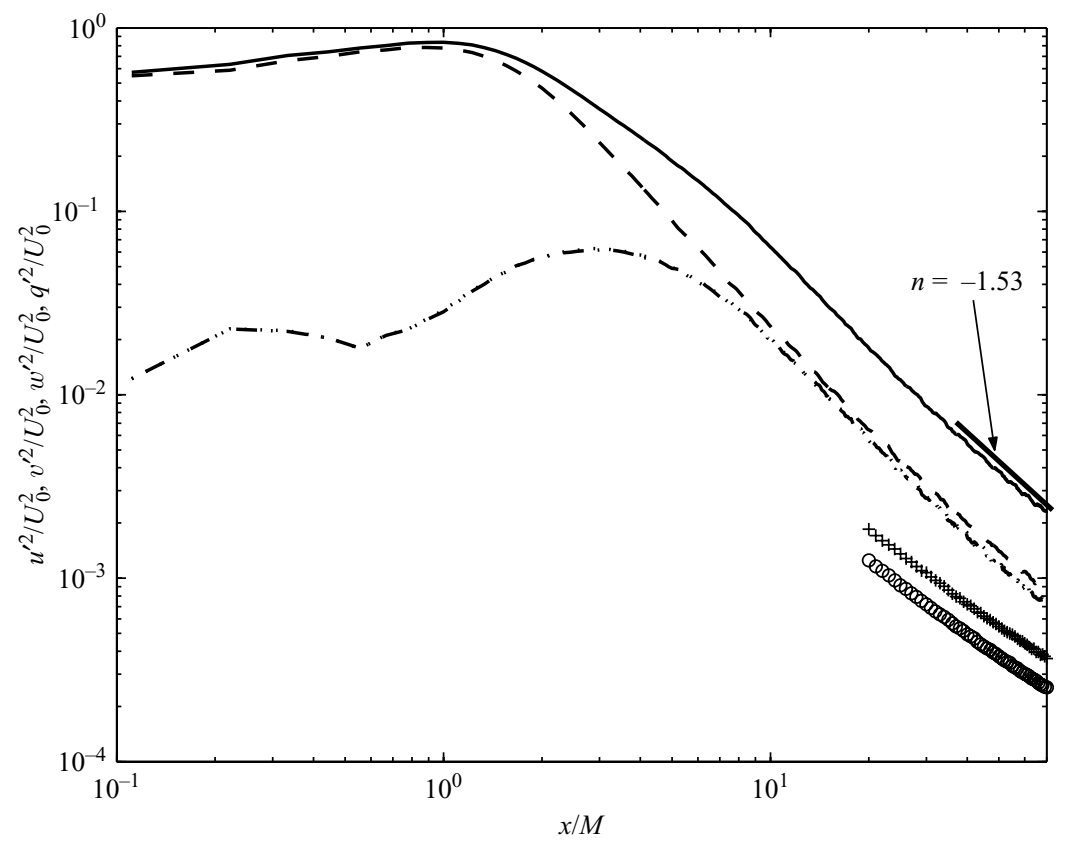

Figure 8. Decay of the turbulent kinetic energy (solid line) and its components (- - $u^{\prime 2} ;-\cdot-, v^{\prime 2} ; \cdots, w^{\prime 2}$ ) downstream of the grid. Symbols: experiments (Lavoie et al. 2005), $\times, u^{\prime 2} ; \bigcirc, v^{\prime 2}$.

makes the curve fitting with a straight line rather inaccurate, which may yield an erroneous value of $n$. To determine whether a straight line region does exist, the function $\operatorname{d} \log \left(q^{\prime 2}\right) / \operatorname{dlog}(x)$ (that is equal to the exponent $n$ ) was plotted (not shown here) between $x / M=50$ and 70 . It was found that $n$ appeared to increase; the variation is from -1.67 at $x / M=50$ to -1.27 at $x / M=70$. It is important to note that while no real attempt was made to determine an effective origin, $x_{0} / M$, required to properly estimate the power-law exponent, it was observed that the variation of $n$ could be reduced by setting $x_{0} / M$ to different values; when $x_{0} / M=-1.5$ (the selection of this particular value is explained later), $n$ varied between -1.5 at $x / M=40$ and -1.25 at $x / M=70$. Common values of $n$ found in the literature range between -1.15 and -1.35 (Mohamed \& LaRue 1990). Several causes can simultaneously affect $n$. The relatively low value of the Reynolds number $R_{M}$, the grid solidity (the present one, $25 \%$, is smaller than that found in the literature, $30 \%$ to $45 \%$ ), the grid geometry, the estimate of $x_{0} / M$ (this is perhaps the most critical factor; on the effects of the virtual origin on $n$ (see also Mohamed \& LaRue 1990), and the possibility that an equilibrium similarity has not been reached, may all contribute to produce different $n$ between different sets of data of grid-generated turbulence. The relatively small number of grid elements may also affect the decay rate. Since the work of Corrsin and co-workers, it is accepted that energy of grid-generated turbulence does not decay correctly unless there are enough grid elements, typically 20 or more across the flow. George et al. (2001) argued that 'crowding turbulence into too small a space (physically and computationally) will result in a decay rate higher than in a corresponding homogeneous turbulence'. This may be the case in the present simulation and further simulation with more grid elements should be carried out. 


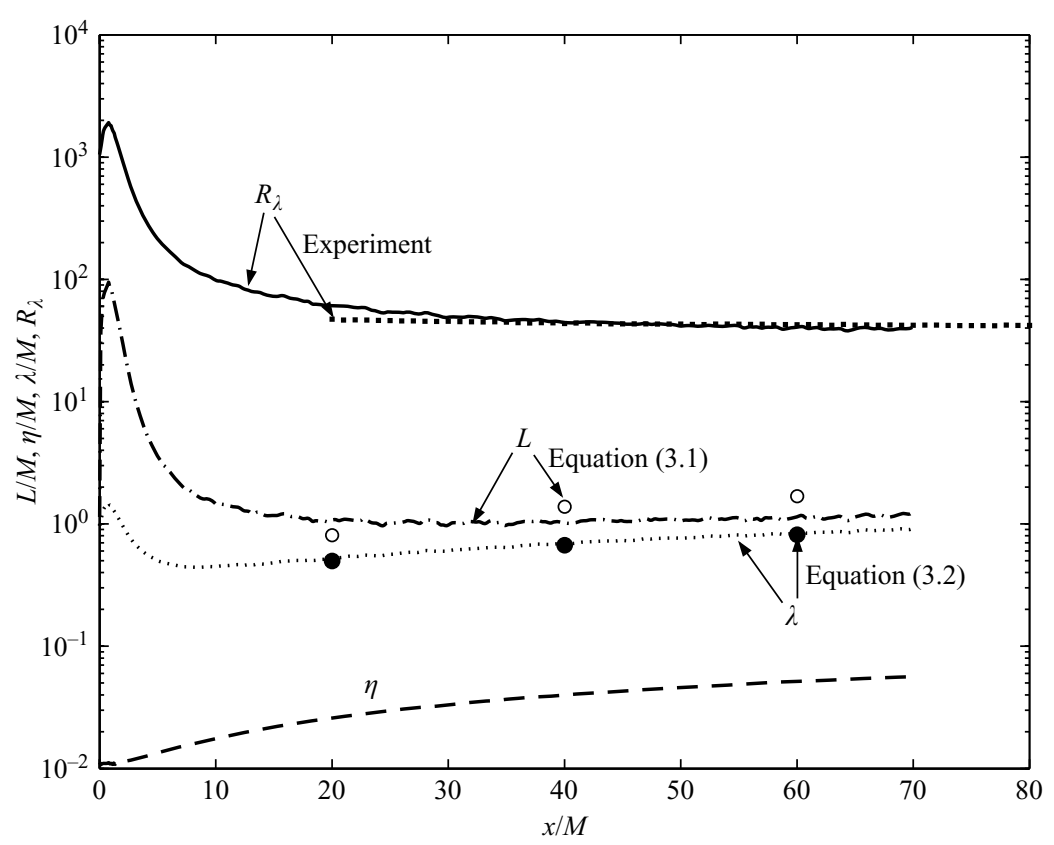

FiguRE 9. Variations of $L(-\cdot-), R_{\lambda}(-) \lambda(\cdots)$ and $\eta(---)$ downstream of the grid. $\bigcirc$, equation (3.1); $\bullet$, equation (3.2); the small filled squares are the $R_{\lambda}$ data of Lavoie et al. (2005).

The ratio $u^{\prime 2} / v^{\prime 2}$ for $x / M>20$ is less than 1.2 , which is below the range of the values found in the literature for grids made of bars (this ratio is about 1.45 for the data of Lavoie et al. 2005a). Again the reasons mentioned above could explain this difference.

The streamwise variation of the integral length scale, $L$, Taylor and Kolmogorov microscales ( $\lambda$ and $\eta$, respectively) and the Reynolds number $R_{\lambda}$ are computed and presented in figure 9 (the length scales are normalized by $M$ ). The $R_{\lambda}$ experimental data of Lavoie et al. (2005) are included for comparison. Here $\lambda$ is equal to $\lambda_{u}$, the Taylor microscale based on the longitudinal velocity fluctuation. Both $L$ and $\lambda$ were computed using their definitions

$$
\begin{aligned}
L & =\int_{0}^{\infty} B_{u u}(r) \mathrm{d} r, \\
\frac{1}{\lambda^{2}} & =\frac{1}{2}\left(\frac{\mathrm{d}^{2} f}{\mathrm{~d} r^{2}}\right)_{r=0},
\end{aligned}
$$

and the isotropic relations for $L$ and $\lambda$

$$
\begin{gathered}
L_{i s o}=\lambda \frac{R_{\lambda}}{30}, \\
\frac{1}{\lambda^{2}}=\frac{1}{2 u^{\prime 2}} \overline{\left(\frac{\mathrm{d} u}{\mathrm{~d} r}\right)_{r=0}^{2}} .
\end{gathered}
$$

In the above equations, $B_{u u}(r)=\langle u(x) u(x+r\rangle) / u^{\prime 2}, r$ is the streamwise separation, and $f=B_{u u}(r)$ is the longitudinal velocity autocorrelation function; the brackets $\langle$. 


$\begin{array}{lccc} & x / M=20 & x / M=40 & x / M=60 \\ L_{y} / L & 10 & 5.84 & 4.8 \\ L / \Delta x & 7.25 & 12.5 & 15.13 \\ \lambda / \Delta x & 4.5 & 6.05 & 7.34 \\ \eta / \Delta x & 0.23 & 0.38 & 0.46 \\ R_{\lambda} & 61.2 & 44.6 & 40.6\end{array}$

TABLE 1. Ratios $L / \Delta x$ and $\eta / \Delta x$ and $R_{\lambda} ; L_{y}$ is the lateral size of the computational domain.

denotes ensemble averaging and the overbar time averaging. Gamard \& George (2000) called the length scale $L_{i s o}$ in equation (3.3) the pseudo-integral scale (it is defined as $u^{\prime 3} / \epsilon$ ). They argued that only in the limit of an infinite Reynolds number is this scale proportional to $L$.

Once turbulence is generated, it decays in a typical fashion: the Reynolds number decreases $\left(R_{\lambda}=40\right.$ at $\left.x / M=70\right)$ and the turbulence length scales $(L, \lambda$ and $\eta)$ increase; the values of $L / M, \lambda / M$ and $\eta / M$ vary from $0.8,0.5$ and 0.027 at $x / M=20$ to 1.67 , 0.8 and 0.05 at $x / M=60$, respectively. The relatively high values of $R_{\lambda}$ just behind the grid reflect the high energy transfer resulting from the interactions between the vortical structures shed by the grid elements (as seen in §3.1). Of course, due to their interaction these turbulence-generating large structures lose energy and generate smaller structures, hence the reduction of $R_{\lambda}$ as $x / M$ increases.

Interestingly, while both expressions for $\lambda$, (3.2) and (3.4), yield virtually the same values, the integral length scale $L$ obtained with its definition (3.1) differs markedly from $L_{i s o}$. This is exactly as Gamard \& George (2000) have pointed out, and is a consequence of the low Reynolds number and the absence of an inertial subrange in the present data (see the spectra plots in figures 16-19). To discuss this difference, ratios $L_{y} / L$ ( $L_{y}$ is the lateral length of the computational domain), $L / \Delta x, \eta / \Delta x$ and $R_{\lambda}$ for $x / M=20,40$ and 60 are reported in table 1 . The table shows that the ratio of the lateral size of the computational domain to $L$ decreases by a factor two when $x / M$ increases from 20 to 60 ; at $x / M=40$ this ratio is already down to about 6 . Note that while at $x / M=20, L_{y}$ is about $10 L$, which may be enough to resolve for the large scales, $\lambda$ is only about 4 times the spatial step $\Delta x$, which is certainly not enough to properly capture the small-scale motions. In that respect it is not surprising that (3.1) does not agree well with (3.3). The drop in the ratio $L_{y} / L$ is unfortunately accompanied by poorer resolution of the large-scale motions which are associated with turbulence production. This in turn may imply that the large-scale motions may not be adequately simulated or even be missing from the solution, possibly preventing a proper estimate of the integral length scale $L$. This would corroborate Wang \& George's (2002) discusion on how the missing of the large-scale motions affect the energy and intergral scales.

To discuss further the problem of missing scales, the ratios $L / \lambda$ and $\lambda / \eta$ are reported in figure 10. Since expressions (3.2) and (3.4) yield the same value of $\lambda$ (see figure 9), equation (3.4) was used for convenience. Expression (3.1) was used for calculating $L$. While both the isotropic $\left((\lambda / \eta)_{i s o}=30^{1 / 4} R_{\lambda}^{1 / 4}\right)$ and the computed values of $\lambda / \eta$ show consistency in both their values (at $x / M=60, \lambda / \eta=1.09(\lambda / \eta)_{i s o}$ ) and their trend, the same is not observed for $L / \lambda$. The computed ratio $L / \lambda$ increases between $x / M=20$ and 40 then starts a slight decrease (not visible in the present scale). Also the magnitude of $L / \lambda$ and $(L / \lambda)_{i s o}\left(L / \lambda=1.54(L / \lambda)_{i s o}\right)$ differs noticeably. 


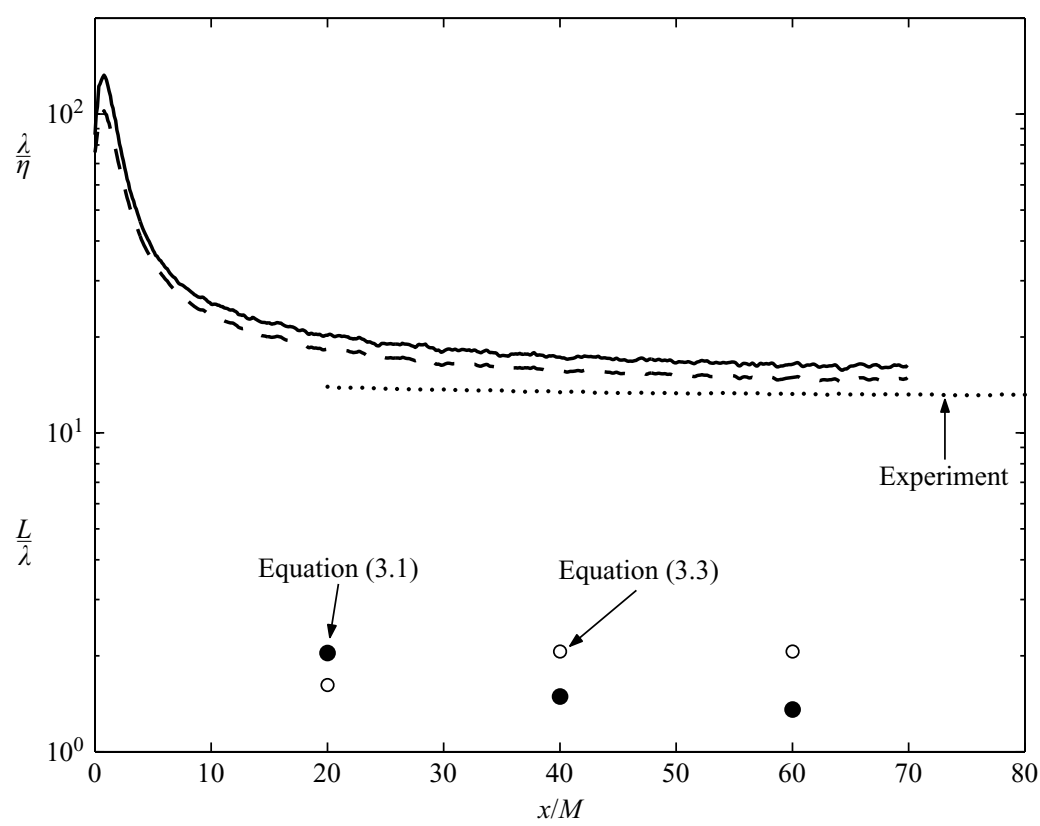

FIGURE 10. Variations of $L / \lambda$ and $\lambda / \eta$ downstream of the grid. Dashed line: present calculation, solid line: $\left.(\lambda / \eta)_{\text {iso }}=30^{1 / 4} R_{\lambda}^{1 / 4}\right)$; $\bullet$, equation (3.1); O, equation (3.3); the small filled circles are the $\lambda / \eta$ data of Lavoie et al. (2005).

This observation tends to confirm that the large scales do suffer from boundary condition effects (i.e. the limits of the lateral size of the computational domain). On the other hand, the smaller length scales appear to be less influenced by such constraints. Furthermore, the resolution of these small scales increases with $x / M$ as is shown in table 1.

The seemingly constant value of $L / \lambda$ has to be treated with caution. It is commonly thought that this ratio decreases as $x / M$ increases unless $R_{\lambda}$ remains contant. George's (1992) analysis suggests that the ratio remains constant. Unfortunately, the present data cannot provide a definitive conclusion, since $L$ is not properly resolved. More extensive simulation with a larger computational domain should be carried out.

The statement that the small-scale motions may be resolved can be checked with $\lambda$. A requirement for a power decay is, for example,

$$
\frac{q^{\prime 2}}{U_{0}^{2}}=A\left(\frac{x}{M}-\frac{x_{0}}{M}\right)^{n}
$$

where $A$ is a constant of proportionality and $n$ is constant. Since for isotropic turbulence,

it follows that

$$
\epsilon=-\frac{1}{2} U_{0} \frac{\mathrm{d} q^{\prime 2}}{\mathrm{~d} x}=10 v \frac{q^{\prime 2}}{\lambda^{2}}
$$

$$
\lambda^{2}=-\frac{20 v M}{n U_{0}}\left(\frac{x}{M}-\frac{x_{0}}{M}\right),
$$

The value of $n$ can be inferred from the plot of $\mathrm{d} \lambda^{2} / \mathrm{d} x$, which should be a constant and is shown in figure 11. If one assumes that, within a small variation $( \pm 5 \%), \mathrm{d} \lambda^{2} / \mathrm{d} x$ 

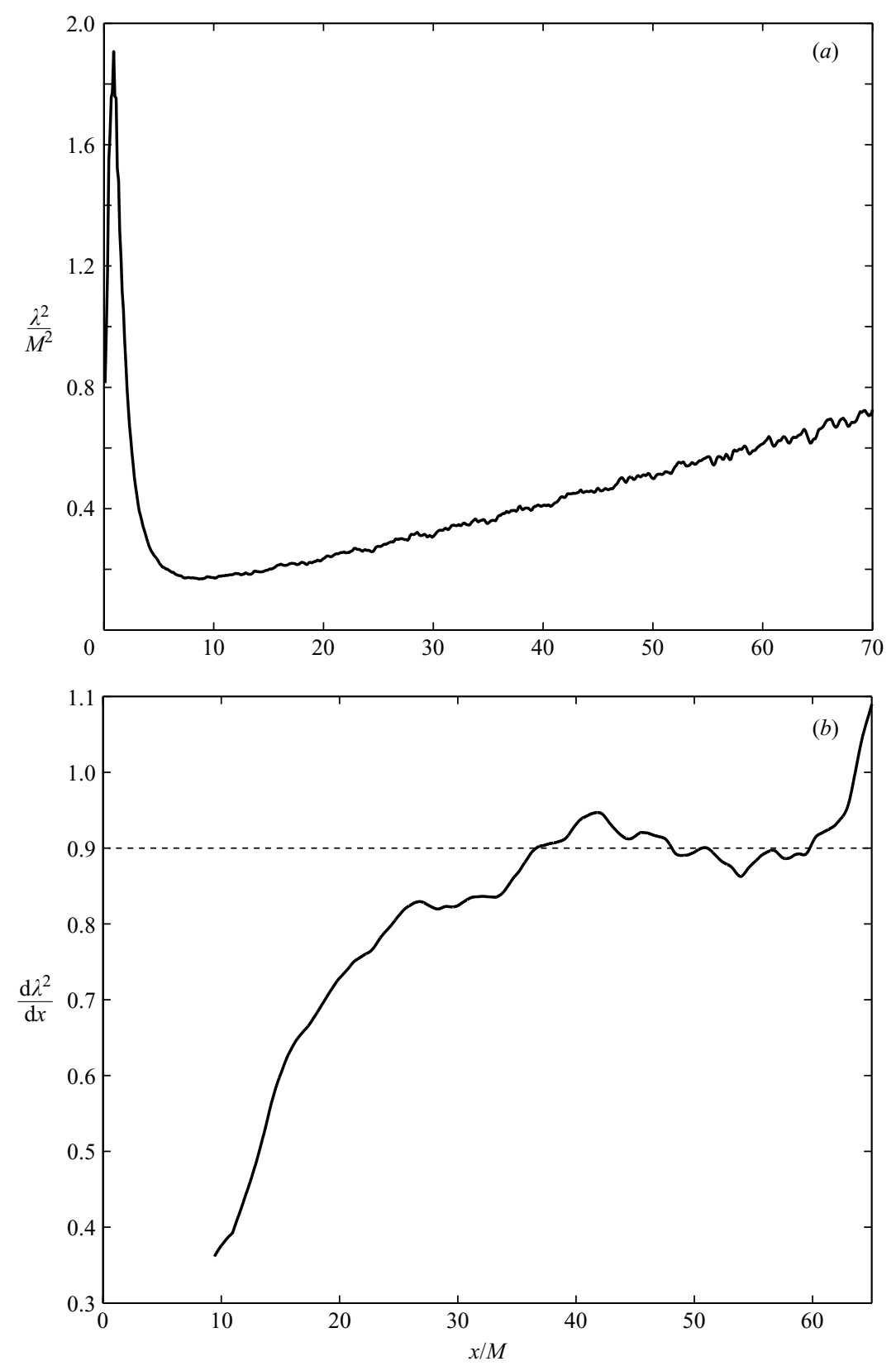

FIGURE 11. Dependence of $\lambda^{2} / M^{2}$ and $\mathrm{d} \lambda^{2} / \mathrm{d} x$ on $x / M$.

presents a plateau over a limited region $(40<x / M<60$; see figure $11 b)$, the inferred value of $n$ is -1.12 . It is interesting to note that this value is close to that $(-1.17)$ for the DNS data of deBruyn Kops \& Riley (1998), once corrected for the missing large-scale motion (Wang \& George 2002). This value of $n$ yields an estimate of $x_{0} / M$ of about -1.5 . Using this in (3.5) produces an exponent value $n$ of about -1.3 , which is in contrast with the value of -1.53 originally obtained, but still quite different from $-1,12$. The fact that the value of $n$ obtained with (3.7) differs from that estimated 


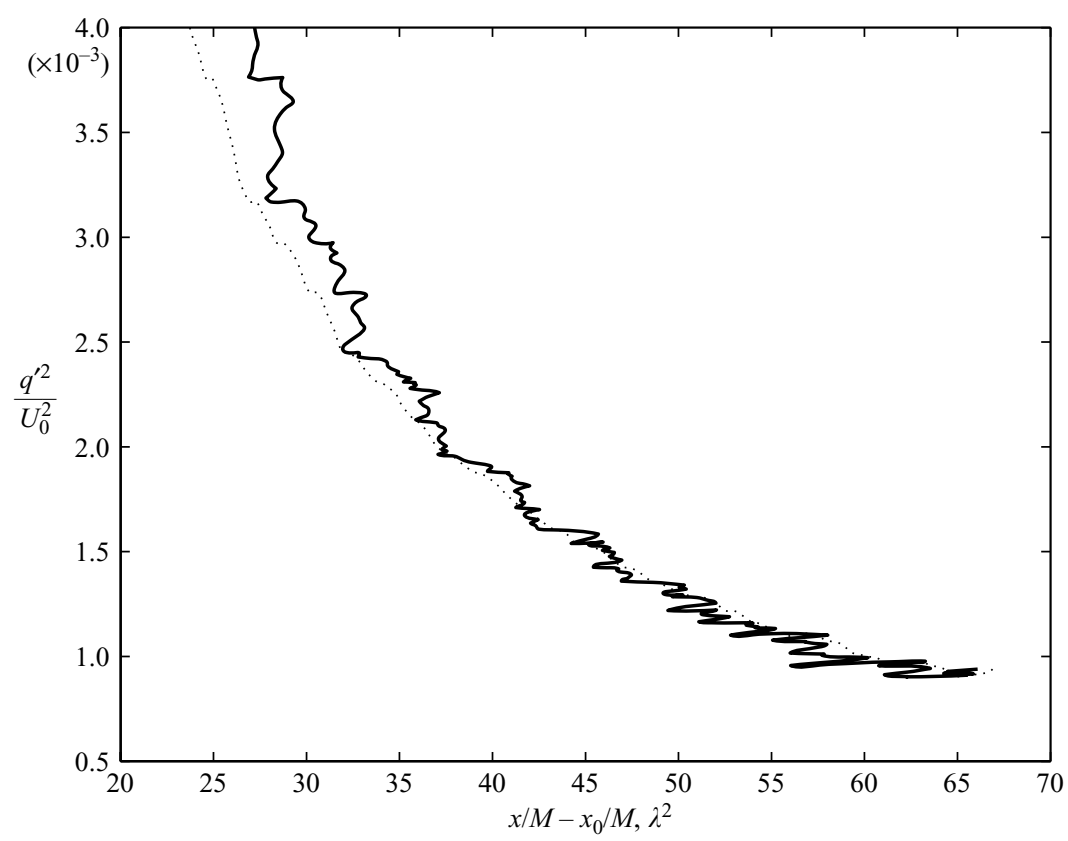

FIGURE 12. Decay of $q^{\prime 2} / U_{0}^{2}$ as a function of $\left(x / M-x_{0} / M\right)$ (dotted line) and $\lambda^{2}$ (solid line).

with (3.5) highlights one particular problem that simulations as well as experiements may face. The problem is related to the assumptions that a power law does exist and $n$ is indeed constant. The former assumtion may prove to be critical in some instances. For example, while figure 11(a) would suggest that $\lambda^{2} / M^{2}$ is proportinal to $x / M$, figure $11(b)$ clearly shows that it is not strictly true. This observation illustrates vividly the difficulty one can face in assessing the decay of isotropic turbulence. In particular, it points to the need for caution when fitting a power law to data which, for various reasons, may not reflect the true decaying state of isotropic turbulence.

To illustrate that one can easily draw wrong conclusions on the decay exponent $n$, another way to assess the existence of a power-law decay for isotropic turbulence, which does not require knowledge of $x_{0} / M$, is presented next. If a truly isotropic turbulence decays as a power law, then both

$$
\lambda^{2}=-B\left(\frac{x}{M}-\frac{x_{0}}{M}\right)
$$

where $B$ is a constant of proportionality, and expression (3.5) are valid simultaneously. One can then write

$$
\frac{q^{\prime 2}}{U_{0}^{2}}=C \lambda^{2 n}
$$

with $C$ a constant (its unit is such that the ratio is dimensionless). Not only should the exponent $n$ be deducible from a log-log plot, but also a region satisfying a power-law decay should be visible, if indeed such a region does exist for the present computation. Figure 12 shows $q^{\prime 2} / U_{0}^{2}$ versus both $\left(x / M-x_{0} / M\right)$ and $\lambda^{2}$ in a log-log scale. The value -1.5 for $x_{0} / M$ was used for consistency between expressions (3.5) and (3.9). A region where both expressions (3.5) and (3.9) would seem to be compatible may be perceptible for $\left(x / M-x_{0} / M\right)>50$, suggesting the possible existence of a power-law decay region. However, it was found above that (3.5) does not yield the same $n$ value 


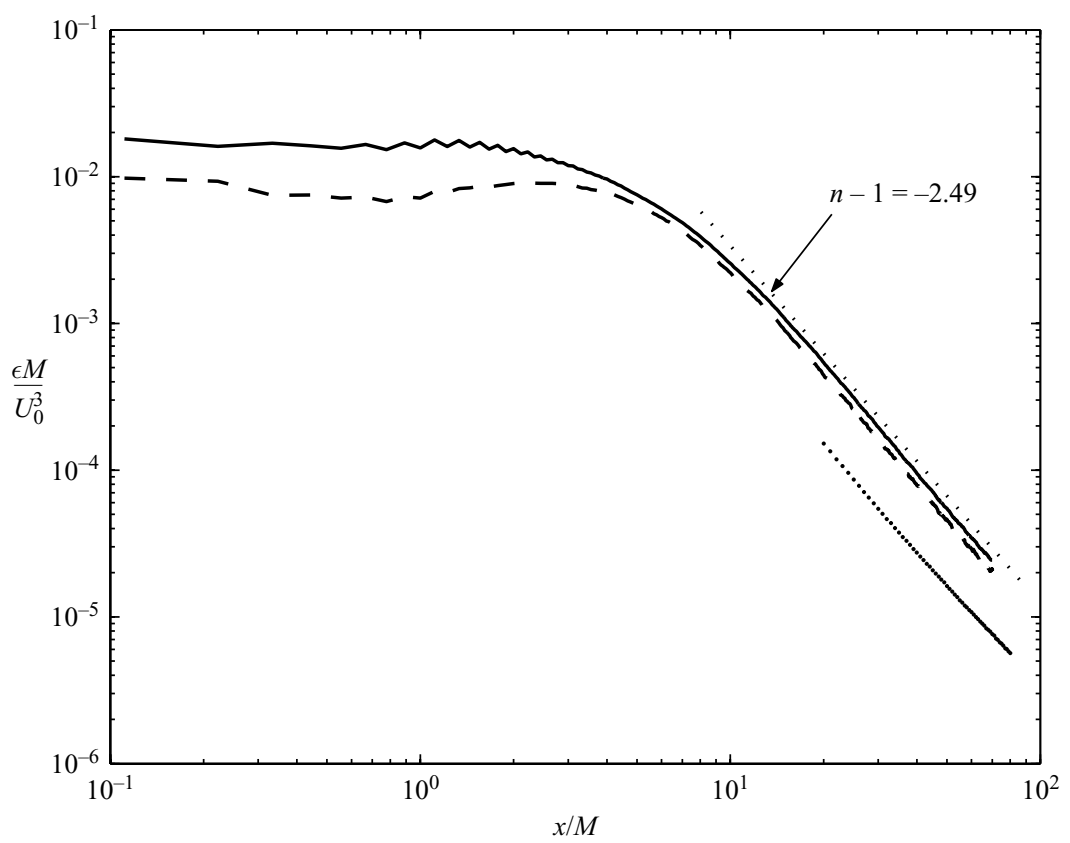

FIGURE 13. Decay of the turbulent kinetic energy dissipation rate, $\epsilon$. Solid line: $\epsilon_{\text {true }}$, dashed line: $\epsilon_{i s o}$; symbols: experiment (Lavoie et al. 2005).

as (3.7). This suggests that either a power law is not well-satisfied or only on a very limited range; both cases make the determination of $n$ difficult. The reasons for this, at least for the present simulation, are likely to be related to the mesh resolution and computational size effects, which would corroborate the arguments of Wang \& George (2002).

Quite remarkably, the decay of the turbulent kinetic energy dissipation rate,

$$
\epsilon_{\text {true }}=\overline{v \frac{\partial u_{i}}{\partial x_{j}}\left(\frac{\partial u_{i}}{\partial x_{j}}+\frac{\partial u_{j}}{\partial x_{i}}\right)}
$$

(figure 13) appears to be consistent with the decay of $q^{\prime 2} / U_{0}^{2}$, i.e. $\epsilon \propto\left(x / M-x_{0} / M\right)^{n_{\epsilon}}$. The dissipation power-law decay exponent $n_{\epsilon}$ is estimated to be -2.40 (when $x_{0} / M$ is set to -1.5$)$, yielding a value of $n\left(=n_{\epsilon}-1\right)$ of -1.40 , not very different to the value -1.3 obtained from (3.5) (when $x_{0} / M=-1.5$ ). The locally isotropic value of $\epsilon\left(\epsilon_{i s o}=30 \nu u^{\prime 2} / \lambda^{2}\right)$, is also shown for comparison. It is quite encouraging to note that the computed $\epsilon_{\text {true }}$ follows the theoretical distribution $\epsilon_{i s o}$; this adds some confidence to the present simulation, at least for the solution of the small scales: it indicates that the present grid resolution is satisfactory for calculating $\epsilon$. However, as expected and already observed, isotropy is not satisfied. Not surprising too, the computed values of $\epsilon$ are higher than the experimental data, which is consistent with the data of figure 8 .

Figure 14 shows the $x$-variations of the velocity derivative skewness, $S$, flatness, $F$, and the product $S R_{\lambda}$. Results from the experiment of Lavoie et al. (2005) are also shown. At $x / M=60, F=3.35$, and $S=-0.251$; for the experiment, these values are 4.2 and -0.47 , respectively. Notice that the seemingly constant value of the product $S R_{\lambda}$ is consistent with an apparant increase of $S$ and decrease of $R_{\lambda}$. This would be in agreement with George's (1992) theory but would contradict measurements (as for example seen in the data of Lavoie et al.). Also, direct numerical simulations 


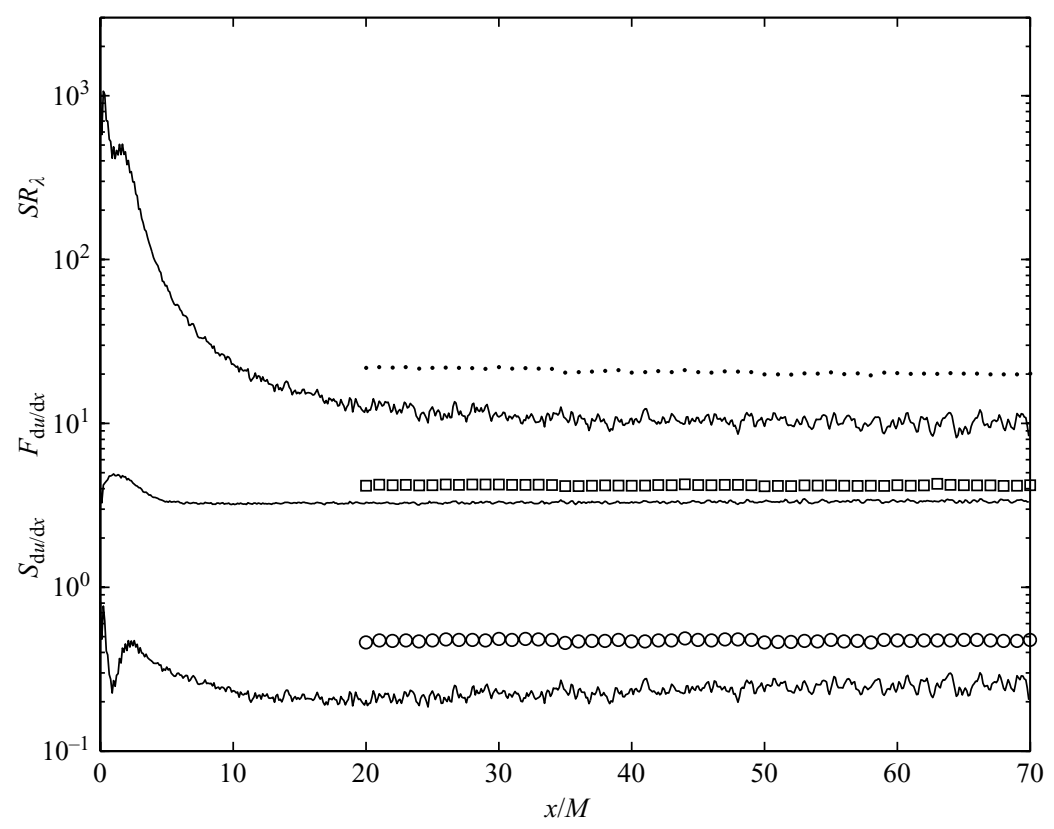

FIGURE 14 . Velocity derivative skewness, $S$, flatness, $F$, and product $S R_{\lambda}$. Lines: simulation, symbols: experiment (Lavoie et al. 2005).

of decaying homogeneous and isotropic turbulence in a periodic box (Wray 1998; Antonia \& Orlandi 2004) and measurements in grid turbulence (Zhou et al. 2000) indicate that $S$ reaches a constant value (about -0.55 ). Since the present mesh resolution $\Delta x$ is only, at best, $2 \eta$, and the grid resolution requirements for a good estimate of the velocity derivative skewness are much more severe than those for the estimates of $\lambda$ and $\epsilon$, more simulations are required at a finer resolution (say $\Delta x=0.25 \eta$ ) to discuss further this rather controversial issue.

An important consequence of the relatively small values of $R_{\lambda}$ in the region $20<x / M<60$ is the non-existent inertial range, as seen in figure 15 showing the second-order streamwise velocity structure function $\delta u^{2}$ at $x / M=20,40$ and 60 . Here

$$
\delta u^{2}=\left\langle u^{2}(x+r)-u^{2}(x)\right\rangle .
$$

Also shown are $\delta u^{2}$ and $\delta q^{2}$ computed from the time series (at $x / M=60$; Taylor's hypothesis, $r=U \Delta t$, was used to convert time separation into space separation) and $\delta q^{2}$ of Lavoie et al. (2005). The $r^{2 / 3}$ straight line represents the inertial range. The computed data do not show a visible inertial region. Note that the data tend to collapse in the dissipative range characterized by the slope 2 . As already mentioned, the grid resolution is not fine enough to resolve the scales $r \leqslant \eta$. The shift observed in the data for $x / M=20$ is likely to result from the relatively poor grid resolution at this location. Notice that from the time series the smallest separation is $r / \eta=0.1$. This is because, while the mesh resolution is larger than $\eta$, the simulation time step $(\Delta t=1$ in the time lattice unit) is much smaller than the Kolmogorov time scale $\left(\tau_{K}=750 \Delta t\right.$ at $x / M=60$ ). Remarkably, $\delta u^{2}$ and $\delta q^{2}$ follow the $r^{2}$ law well.

Perhaps the best way to assess the effects of the grid resolution on the simulation, at both large and small scales, is through the spectra. Figure 16 and figure 17 show the one-dimensional energy spectra, $E(k \eta)$, and the longitudinal one-dimensional spectra, $E_{11}(k \eta)$, at $x / M=20,40$ and 60 , where $k$ is the longitudinal wavenumber $k=2 \pi f / U_{0}$. 


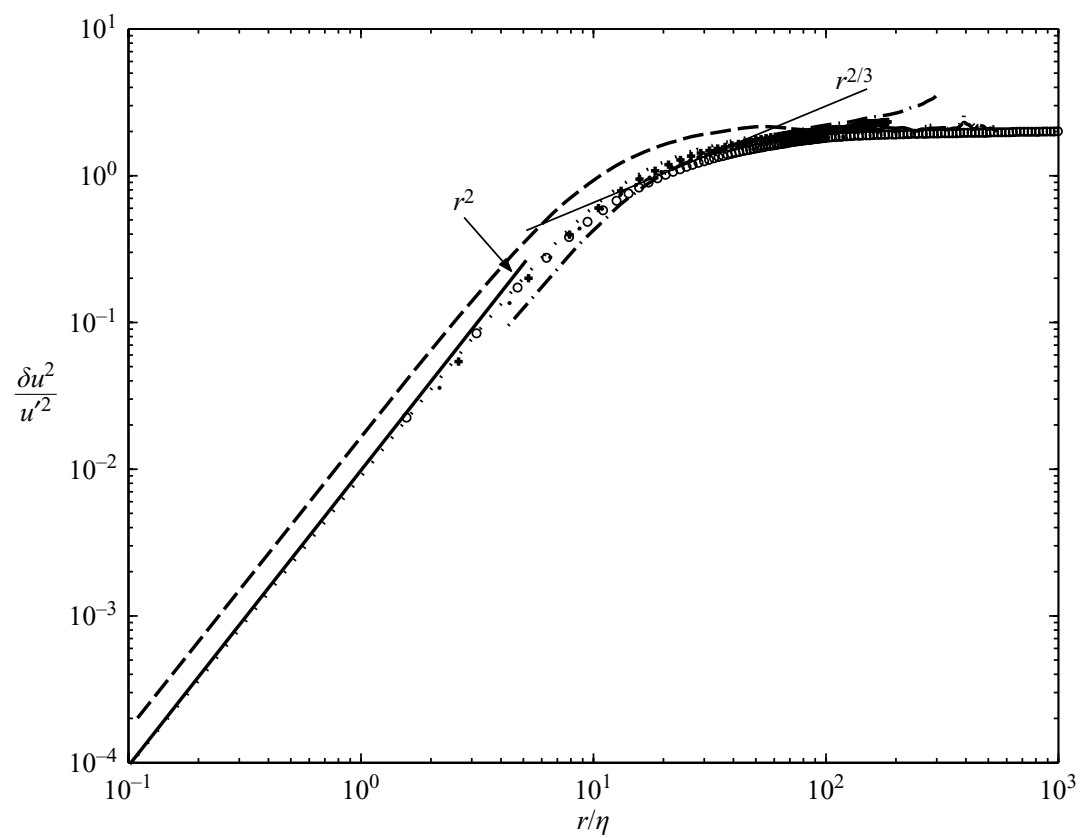

FIGURE 15. Second-order structure function of the streamwise velocity. Lines: simulation, symbols: experiment (Lavoie et al. 2005). $x / M=20(-\cdot-) ; x / M=40(+) ; x / M=60(\bullet)$. Also shown are $\delta q^{2} / q^{\prime 2}(--)$ and $\delta u^{2} / u^{\prime 2}(\cdots)$ calculated from the time series using Taylor's hypothesis $\bigcirc, \delta u^{2} / u^{\prime 2}$ of Lavoie et al. (2005).

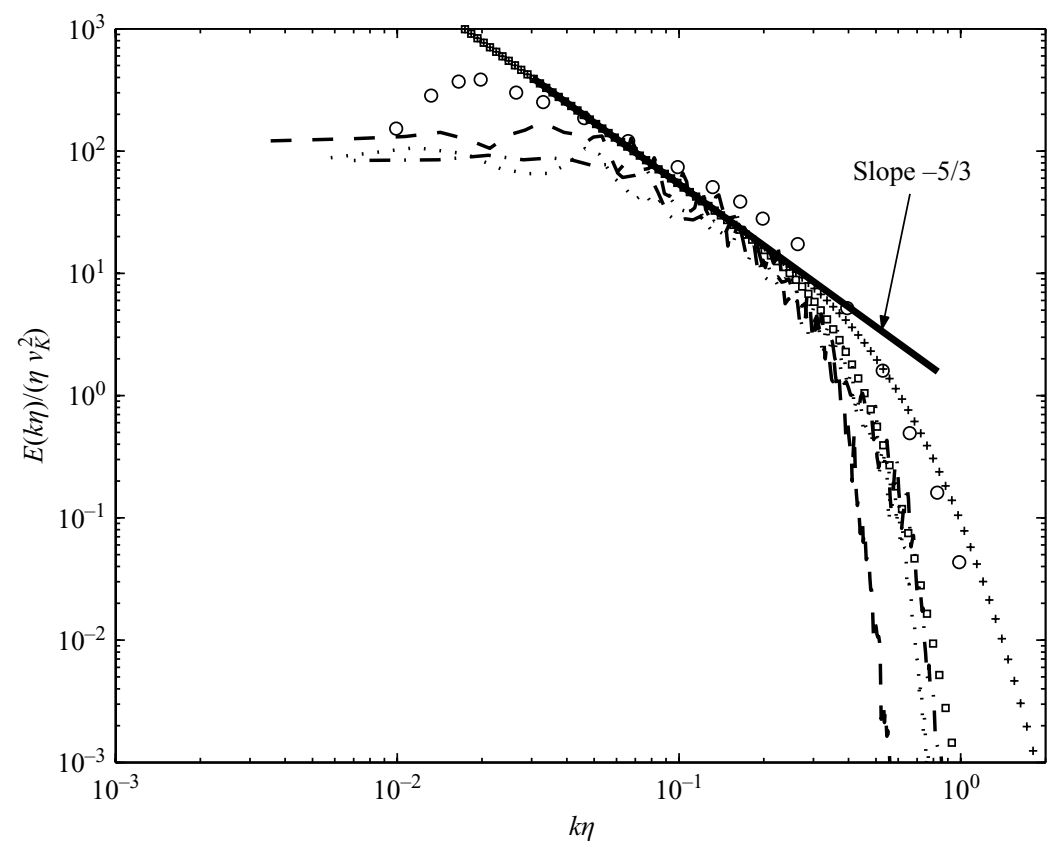

FIGURE 16. One dimensional energy spectra $E(k)$ in Kolmogorov units at $x / M=20(---)$, $40(\cdots)$ and $60(-\cdot-)$. O, experiment (Comte-Bellot \& Corrsin 1971); small squares and crosses: equation (3.11) with $\beta=3.16$ and 12.84 , respectively. 


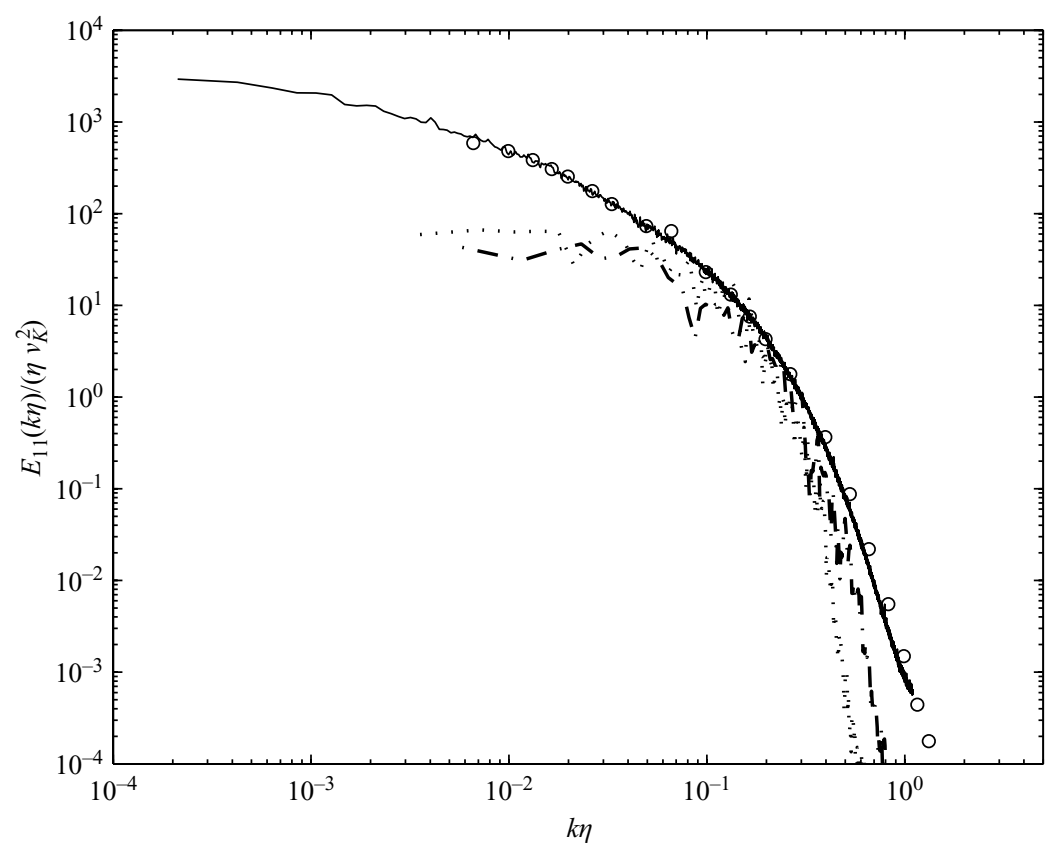

FIGURE 17. Longitudinal one dimensional spectra $E_{11}(k)$ in Kolmogorov units at $x / M=$ $20(\cdots)$ and $60(-\cdot)$. - , experiment of Lavoie et al. (2005), symbols: experiment of Comte-Bellot \& Corrsin (1971).

Also shown are $E(k \eta)$ and $E_{11}(k \eta)$ of Comte Bellot \& Corrsin $\left(1971, U_{0} t / M=171\right)$ and $E_{11}(k \eta)$ of Lavoie et al. 2005). The theoretical energy spectra of Pope (2000) for high wavenumbers are also included:

$$
E(k \eta / \eta) v_{K}^{2}=C(k \eta)^{-5 / 3} f_{\eta}(k \eta)
$$

where

$$
f_{\eta}(k \eta)=\exp \left\{\left(-\beta\left\{\left[(k \eta)^{4}+c_{\eta}^{4}\right]^{1 / 4}-c_{\eta}\right\}\right)\right\}
$$

$v_{K}$ is the Kolmogorov velocity, $C=1.16, c_{\eta}=0.4$ and $\beta=3.16$ and 12.84 . The values of $C$ and $\beta$ differ from those given by Pope to fit the present results.

While the calculated spectra present similar features to those observed in the experiment, several comments are nevertheless required. First, as expected the inertial region is non-existent in the calculated spectra. Secondly, the spectrum at $x / M=20$ differs from those at $x / M=40$ and 60 at both ends of the spectrum. This deviation reflects mainly two effects: (i) low grid resolution and (ii) finite limits of the lateral sizes of the computation. This is well-illustrated in table 1 shown earlier. It was observed that as the distance $x / M$ increases the grid resolution improves for the small scales, but deteriorates for the large scales.

Thus, at $x / M=20$, the resolution is better for the low wavenumbers, but poorer at $x / M=40$ and 60 in the high wavenumbers. The relatively strong drop in the spectrum for $x / M=20$ is due to the LES scheme. Indeed, the dissipation induced by the LES is felt early in the spectrum at this longitudinal location because of the relatively poor grid resolution. At $x / M=40$ and 60 , the improved grid resolution yields better spectra in the high-wavenumber region. On the other hand, the finite limits of the lateral sizes of the computation affect the simulation at these stations, which contaminates the resolution of the large scales. 


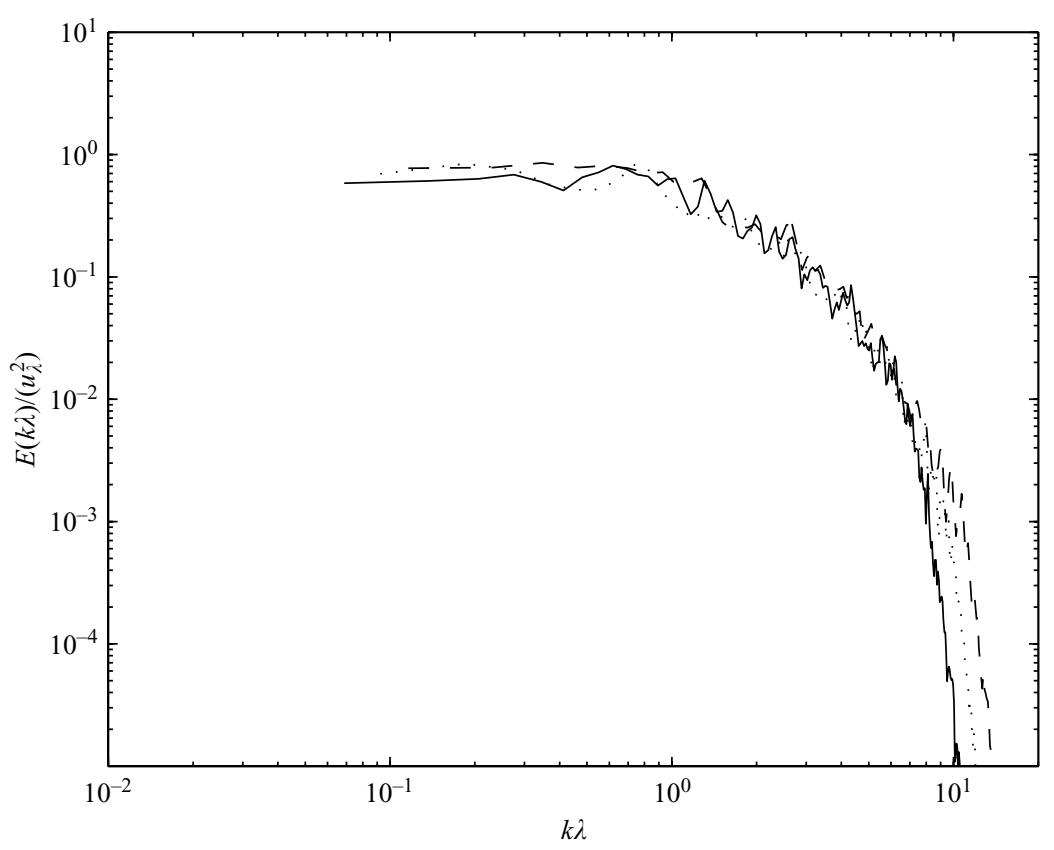

FIGURE 18. One dimensional energy spectra $E(k)$ normalized by $u^{2} \lambda$ at $x / M=20(-)$, $40(\cdots)$ and $60(-\cdot-)$.

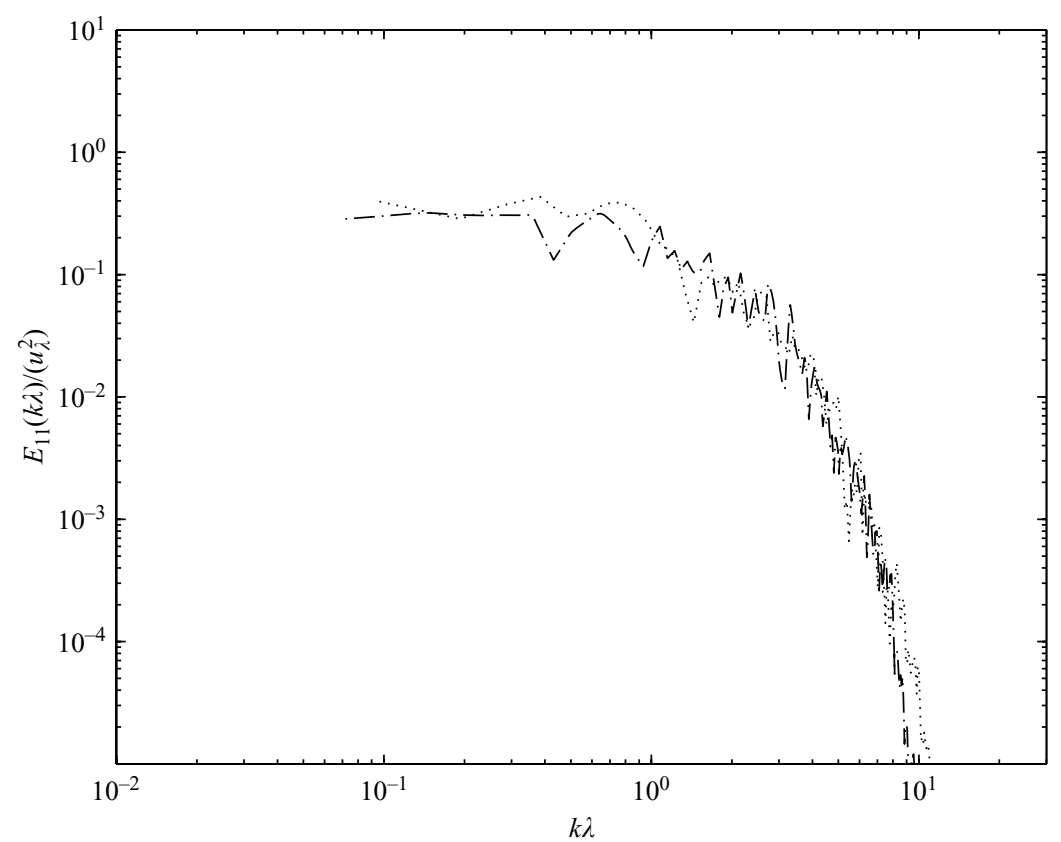

FIGURE 19. Longitudinal one dimensional spectra $E_{11}(k)$ normalized by $u^{2} \lambda$ at $x / M=20$ $(-\cdot)$ and $60(\cdots)$.

The effects of the resolution of the smallest scales on the simulation can be further assessed by plotting $E(k)$ and $E_{11}(k)$ normalized with $\lambda$ and $u^{\prime 2}$ (Taylor's variables). Figures 18 and 19 show the spectra thus normalized. A good collapse is observed 
over the resolved scales of the spectra. George (1992) reported a similar collapse in the experiemental data of Comte-Bellot \& Corrsin (1966) and the DNS results of Antonia \& Orlandi (2004). Apart from the fact that the collapse may be consistent with a self-preserving state of the decaying turbulence (George 1992), it indicates that the limitation of the grid resolution for the small scales is not too critical for the present simulation.

\section{Concluding remarks}

A direct numerical simulation of grid-generated turbulence has been carried out using the lattice-Boltzmann method. The turbulence-generating grid was made of floating square elements, yielding a solidity of 0.25 . The simulation reproduced quite well the results obtained in grid turbulence generated in laboratory. Furthermore, it illustrated quite vividly the major problems that both numerical and experimental studies can have in the study of the decay of (approximately) homogeneous isotropic turbulence. For example, it was found that extra care should be taken when one tries to assess the existence of a power-law decay. In that regard, the results support the view (see Wang \& George 2002) that the best objective method for this assessment is to determine if $\mathrm{d} \lambda^{2} / \mathrm{d} x$ is constant; the value of this constant yields the powerlaw decay exponent unambiguously. Indeed, for a power-law decay to exist, $\lambda^{2}$ must increase linearly with $x$ (the downstream distance).

The present LBM simulation showed, at least in the numerical studies, that the limitations of the simulation, namely the mesh resolution and boundary conditions, can yield incorrect or only approximate results. These limitations were clearly visible in the turbuence kinetic energy and the longituginal velocity spectra. By not accounting for the large-scale motion (the computational domain is not large enough in terms of integral length scale) or resolving the small-scale adequately (the mesh step was at best two times the Kolmogorov length scale), both ends of the spectra were poorly solved. This has implication for the decay of turbulence. For example, one may argue that, unless care is taken, fitting of a power law to data may produce results which reflect the limitations of the simulation and not the true decay.

\section{REFERENCES}

Antonia, R. A. \& Orlandi, P. 2004 Similarity of decaying isotropic turbulence with a passive scalar. J. Fluid Mech. 505, 123-151.

Birouk, M., SARH, B. \& GoKalp, I. 2003 An attempt to realise experimental isotropic turbulence at low Reynolds number. Flow, Turbulence Combust. 70, 325-348.

DeBruyn Kops, S. M. \& Riley, J. J. 1998 Direct numerical simulation of laboratory experiments in isotropic turbulence. Phys. Fluids 10, 2125-2127.

Chen, S. \& Doolen, G. D. 1998 Lattice Boltzmann method for fluid flows. Annu. Rev. Fluid Mech. 30, 329-364.

Comte-Bellot, G. \& Corrsin, S. 1966 The use of a contraction to improve the isotropy gridgenerated turbulence, J. Fluid Mech. 25, 657-682.

Comte-Bellot, G. \& Corssin, S. 1971 Simple Eulerian time correlation of full and narrow band velocity signals in grid-generated 'isotropic' turbulence. J. Fluid Mech. 48, 273-337.

Frisch, U., Hasslacher, B. \& Pomeau, Y. 1986 Lattice-gas automata for the Navier-Stokes equations. Phys. Rev. Lett. 56, 1505-1508.

Gamard, S. \& George, W. K. 2000 Reynolds number dependence of energy spectra in the overlap region of isotropic turbulence. J. Flow Turbulence Combust. 63, 443-337.

George, W. K. 1992 The decay of homogeneous isotropic turbulence. Phys. Fluids A 4, 1492-1509. 
George, W. K., Wang, H., Wollblad, C. \& Johanson, T. G. 2001 Homogeneous turbulence and its relation to realizable flows. In Proc. 14th Australasian Fluid Mechanics Conference (AFMC), Adelaide, 10-14 December.

Hou, S., Sterling, J., Chen, S. \& Doolen, G. D. 1996 A lattice subgrid model for high Reynolds number flows. Fields Institute Commun. 6, 151-166.

Hwang, W. \& Eaton, J. K. 2004 Creating homogeneous and isotropic turbulence without mean flow. Exps. Fluids 34, 444-454.

Lavoie, P., Antonia, R. A. \& Djenidi, L. 2006 Effect of initial conditions on the generation of coherent structures in grid turbulence. Whither Turbulence Prediction and Control Conference, Seoul, March, 26-29, 2006. Centre for Turbulence and Flow Control, Seoul National University/Centre for Turbulence Research, Stanford University.

Lavoie, P., Burattini, P., Duenidi, L. \& Antonia, R. A. 2005 Effect of initial conditions on decaying grid turbulence at low $R_{\lambda}$. Exps. Fluids 39, 865-874.

Mohamed, M. S. \& LaRue, J. C. 1990 The decay power law in grid-generated turbulence. J. Fluid Mech. 219, 195-214.

Zhou, T., Antonia, R. A., Danaila, L. \& Anselmet, F. 2000 Transport equations for the mean energy and temperature dissipation rates in grid turbulence. Exps. Fluids 28, 143-151.

WANG, H. \& GEORGE, W. K. 2002 The integral scale in homogeneous isotropic turbulence. J. Fluid Mech. 459, 429-443.

WraY, A. 1998 Decaying isotropic turbulence. In AGARD Advisory Rep. 345, pp. 63-64.

Pope, S. B. 2000 Turbulent Flows. Cambridge University Press. 